\title{
Roles of Fast-Cyclotron and Alfvén-Cyclotron Waves for the Multi-Ion Solar Wind
}

\author{
Ming. Xiong ${ }^{1} \cdot$ Xing. Li $^{1}$.

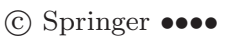

\begin{abstract}
Using linear Vlasov theory of plasma waves and quasi-linear theory of resonant wave-particle interaction, the dispersion relations and the electromagnetic field fluctuations of fast and Alfvén waves are studied for a low-beta multi-ion plasma in the inner corona. Their probable roles in heating and accelerating the solar wind via Landau and cyclotron resonances are quantified. In this paper, we assume that (1) low-frequency Alfvén and fast waves, emanating from the solar surface, have the same spectral shape and the same amplitude of power spectral density (PSD); (2) these waves eventually reach ion cyclotron frequencies due to a turbulence cascade; (3) kinetic wave-particle interaction powers the solar wind. The existence of alpha particles in a dominant proton/electron plasma can trigger linear mode conversion between oblique fast-whistler and hybrid alpha-proton cyclotron waves. The fast-cyclotron waves undergo both alpha and proton cyclotron resonances. The alpha cyclotron resonance in fast-cyclotron waves is much stronger than that in Alfvén-cyclotron waves. For alpha cyclotron resonance, an oblique fast-cyclotron wave has a larger left-handed electric field fluctuation, a smaller wave number, a larger local wave amplitude, and a greater energization capability than a corresponding Alfvén-cyclotron wave at the same wave propagation angle $\theta$, particularly at $80^{\circ}<\theta<90^{\circ}$. When Alfvén-cyclotron or fast-cyclotron waves are present, alpha particles are the chief energy recipient. The transition of preferential energization from alpha particles to protons may be self-modulated by differential speed and temperature anisotropy of alpha particles via the self-consistently evolving wave-particle interaction. Therefore, fast-cyclotron waves as a result of linear mode coupling is a potentially important mechanism for preferential energization of minor ions in the main acceleration region of the solar wind.
\end{abstract}

Keywords: fast wave, linear mode coupling, solar wind

\section{Introduction}

The solar wind is a ubiquitous super-Alfvénic stream of magnetized charged particles from the Sun, consisting of protons, electrons, and minor ions. According

\footnotetext{
1 Aberystwyth University, UK email: mxiong@spaceweather.ac.cn email: xxl@aber.ac.uk
} 
to spectroscopic observations of extreme ultraviolet emission lines in the inner corona, solar wind ions exhibit a large kinetic temperature anisotropy, and minor ions are likely to be preferentially heated and accelerated (Kohl et al., 1998, Li et al., 1998, Cranmer et al., 1999). In the interplanetary space, minor ions have mass-proportional temperatures and similar bulk flow speed, whose drifting speeds relative to protons are at an order of local Alfvén speed (Marsch et al., 1982 Neugebauer et al., 1996: Goldstein et al., 2000). And proton temperature in the direction parallel to the background interplanetary magnetic field is lower than that in the perpendicular direction. Double components of tenuous fast beam and dense core frequently occur in the velocity distribution function (VDF) of protons, with a differential speed of 1.2 - 1.3 Alfvén speed (Marsch et al., 1982). The combination of these remote-sensing and interplanetary in-situ measurements substantiates that some energy should be continuously deposited into the solar wind ions via some physical mechanisms, and thus power the hot collisionless outflow from the Sun.

The solar wind has been an outstanding puzzle for its heating and acceleration since the theoretical prediction (Parker, 1958) and observation verification (Neugebauer and Snyder, 1962). A fraction of the mechanical energy in the Sun's internal convective motions must be converted into thermal energy above the photosphere. Ion cyclotron waves are generally considered as a promising mechanism that can preferentially heat and accelerate minor ions. Ion cyclotron frequencies in the extended corona vary from 10 to $10^{4} \mathrm{~Hz}$, whereas observed frequencies of pronounced oscillation on the solar surface are typically $\approx 0.01 \mathrm{~Hz}$. How strong enough ion cyclotron waves could be generated is still debated. In terms of the origins of ion cyclotron waves, there are two main viewpoints of base and secondary generations. For the base generation, high-frequency Alfvén waves are directly launched during small-scale magnetic reconnection of micro-flares in rapidly evolving super-granular networks, then propagate up through the corona, gradually reach cyclotron frequencies of various ions, and are finally damped over a very short distance (Axford and McKenzie, 1992). For the secondary generation, high-frequency ion cyclotron waves are gradually converted from lowfrequency Alfvén waves in the corona, and such conversion process is probably from a direct turbulence cascade (Li and Habbal, 2001, Markovskii et al., 2010), local nonlinear kinetic instabilities (Markovskii and Hollweg, 2004), and Debyescale electron phase space holes in electron Langmuir turbulence (Cranmer and Ballegooijen, 2003)

In this paper, we assume that (1) the plasma turbulence cascade is the energy source of the solar wind and (2) the solar wind heating is due to Landau and cyclotron resonances with a continuous turbulence spectrum.

The solar wind turbulence is an ensemble of fluctuations with random phases and a broad range of wave vectors. Such fluctuations are manifested in magnetic field $\delta \mathbf{B}$, electric field $\delta \mathbf{E}$, plasma bulk flow velocity $\delta \mathbf{V}$, and so on. The fluctuation energy is usually transferred through a forward cascade. Namely, the energy is injected from a preexisting population of magnetohydrodynamic (MHD) waves at very low frequencies $f$ and wave numbers $k$, nonlinearly transported through successively shorter wavelengths via wave-wave interaction, and eventually dissipated at ion kinetic scales via collisionless wave-particle interaction. The evolution of $\delta \mathbf{B}$ and $\delta \mathbf{V}$ in the presence of a background magnetic 
field $\mathbf{B}_{\mathbf{0}}$ can be represented in the Elsasser variables $\mathbf{Z}^{ \pm}=\delta \mathbf{V} \pm \delta \mathbf{B} / \sqrt{4 \pi \rho_{0}}$ as follows:

$$
\begin{gathered}
\frac{\partial \mathbf{Z}^{ \pm}}{\partial t} \mp\left(v_{\mathrm{A}} \cdot \nabla\right) \mathbf{Z}^{ \pm}+\left(\mathbf{Z}^{\mp} \cdot \nabla\right) \mathbf{Z}^{ \pm}=-\nabla P+F, \\
\begin{cases}k_{\|} B_{0} \gg k_{\perp} b_{\lambda}, & \text { weak turbulence, } \\
k_{\|} B_{0} \approx k_{\perp} b_{\lambda}, & \text { strong turbulence. }\end{cases}
\end{gathered}
$$

Here $v_{\mathrm{A}}=B_{0} / \sqrt{4 \pi \rho_{0}}$ is the Alfvén speed, $\rho_{0}$ is the mass density, $P$ is the pressure that is determined from the incompressibility condition of $\nabla \cdot \mathbf{Z}^{ \pm}=0$, $F$ is a large-scale forcing, $k_{\|}$and $k_{\perp}$ are parallel and perpendicular wave numbers, and $b_{\lambda}\left(\ll B_{0}\right)$ is oscillation amplitude at the scale $\lambda \approx 1 / k_{\perp} . \mathbf{Z}^{ \pm}$is imbalanced at a macro scale, as supported by nonzero cross-helicity in terms of $\delta \mathbf{V} \cdot \delta \mathbf{B}$ (Goldreich and Sridhar, 1995, He et al., 2011). There is more power in Alfvénic fluctuations travelling away from the Sun than towards it. Even if balanced overall, MHD turbulence is always locally imbalanced in creating patches of positive and negative cross-helicities. The conservation of cross-helicity results in a hierarchical structure of MHD turbulence. In the corona, turbulence amplitude is constrained by interplanetary scintillation (IPS) observations of electron density spectra (Cole, 1978). Interplanetary in situ measurements of turbulence $\delta \mathbf{B}$ as a function of $f, k_{\|}$, and $k_{\perp}$ have shown a power law spectrum of magnetic field fluctuation, and a large anisotropy in favor of quasiperpendicular wave propagation (Matthaeus et al., 1990, Leamon et al., 1998, Dasso et al., 2005, Gary and Smith, 2009: MacBride et al., 2010):

$$
\begin{gathered}
|\delta \mathbf{B}(f)|^{2} \approx f^{-\alpha}, \\
\left|\delta \mathbf{B}\left(k_{\|}\right)\right|^{2}=\sum_{k_{\perp}}|\delta \mathbf{B}(\mathbf{k})|^{2} \approx k_{\|}^{-\alpha_{\|}}, \\
\left|\delta \mathbf{B}\left(k_{\perp}\right)\right|^{2}=\sum_{k_{\|}}|\delta \mathbf{B}(\mathbf{k})|^{2} \approx k_{\perp}^{-\alpha_{\perp}}, \\
\alpha_{\|}>\alpha_{\perp} .
\end{gathered}
$$

The solar wind turbulence in an inertial range of $10^{-4} \leq f \leq 0.2 \mathrm{~Hz}$ is similar to the classical Kolmogorov picture of fluid turbulence, and the spectral index $\alpha$ in Equation (3) is approximately 5/3 (Leamon et al., 1998; Horbury et al., 2005). Meanwhile, the power spectral density is anisotropic with respect to $\mathbf{B}_{\mathbf{0}}$, as indicated from $\alpha_{\|} \approx 2$ in Equation (44) and $\alpha_{\perp} \approx 1.6$ in Equation (5) (Gary and Smith, 2009). Moreover, within the inertial range, the Elsasser variables $\mathbf{Z}^{ \pm}$were also found to be anisotropic and have two distinct scaling subranges (Wick et al., 2011). A transition between weak and strong turbulence regimes (Equation (2)) is feasible by changing a large-scale forcing $F$ (Equation (1D)), as demonstrated from numerical simulation of anisotropic incompressible MHD turbulence (Perez and Boldyrev, 2008) 
The nature of the MHD turbulence cascade lies in the interaction of wave packets moving with Alfvén velocities. In the framework of the weak turbulence scenario, interacting wave packets are very large such that the original wave packets decay before they pass through each other. Because the auto-correlation and cascade time scales are always of the same order of magnitude in the weak turbulence theory, any model that implies a large number of collisions among wave packets for an efficient energy cascade should belong to the strong turbulence theory (Gogoberidze et al., 2009). In the strong turbulence, interaction is strong, resonance conditions are not in force, hence a parallel cascade is possible (Perez and Boldyrev, 2008). The forward cascade of $\delta \mathbf{B}$ could be modelled as a combination of advection and diffusion in the wave number $\mathbf{k}$ space (Cranmer and Ballegooijen, 2003). Equations (4) and (5) for turbulence anisotropy in the inertial range could be derived by a conjecture of dynamical alignment that polarizations of $\delta \mathbf{B}$ and $\delta \mathbf{V}$ tend to align during the turbulence cascade towards successively smaller scales (Goldreich and Sridhar, 1995). A good fraction of the power spectral density (PSD) observations is well interpreted by the current turbulence theories.

The turbulence in the dissipation range is poorly understood. Spectral properties in the dissipation range almost certainly depend upon the details of the damping, though those in the inertial range are relatively robust and independent of detailed turbulence cascade processes. The observed wave number at a breakpoint, which separates the inertial and dissipation ranges, may scale as inverse proton inertial length $k c / \omega_{\mathrm{pp}} \approx 1$ (Leamon et al., 1998. Smith et al., 2001) or cyclotron radius $k v_{\mathrm{p}} / \Omega_{\mathrm{p}} \approx 1$ (Bale et al., 2005), with $\omega_{\mathrm{pp}}, \Omega_{\mathrm{p}}$, and $v_{\mathrm{p}}$ denoting proton plasma frequency, proton cyclotron frequency, and proton thermal speed respectively. Above the inertial range, the PSD has a distinct breakpoint at $0.2<f<0.5 \mathrm{~Hz}$; At higher frequencies of $f \geq 0.5 \mathrm{~Hz}$, the PSD is steeper with a broader range of $2 \leq \alpha \leq 4$ in Equation (3) (Leamon et al., 1998; Gary and Smith, 2009). For instance, Sahraoui et al. (2009) found that a PSD above the inertial range consists of two distinct regimes with successively larger values of $\alpha$ in Equation (3); $\alpha$ is about 2.5 from 0.4 to $35 \mathrm{~Hz}$ for a dispersion cascade and 4 from 35 to $100 \mathrm{~Hz}$ due to strong wave damping. Furthermore, it is still unclear what are the constituent wave modes of the solar wind turbulence. The macro-scale fluctuations in the solar wind and corona are Alfvénic in nature (Barnes and Hollweg, 1974). In the MHD regime, Alfvén waves can be converted into fast waves by density fluctuations, and vice versa through resonant three-wave interaction (Chandran, 2008). At the kinetic scale, highly oblique whistler waves exist according to the observed properties of large magnetic compressibility in the solar wind (Gary and Smith, 2009). Kinetic Alfvén waves are identified from the observations of largely enhanced electric fluctuation spectrum and super-Alfvénic wave phase speed at scales of ion thermal gyro-radius (Leamon et al., 1998, Bale et al., 2005). Parallel propagating Alfvén-cyclotron waves are possibly present as evidenced by the measured angle distribution of magnetic helicity (He et al., 2011). These observations have revealed the importance of quasi-perpendicularly propagating waves in the heating of the solar wind.

Increasing attention has been paid to oblique propagation owing to the natural occurrence of oblique waves from refractions in the inhomogeneous solar 
wind, production by density gradients, and generation from MHD turbulence (Li and Habbal, 2001: Gary and Nishimura, 2004: Osmane et al., 2010; Li et al., 2010: Markovskii et al., 2010). Indeed, for the Alfvén-cyclotron wave branch, parallel propagation has its inherent difficulty to reconcile both the heating and the acceleration of the solar wind (Hollweg and Isenberg, 2002). The nature of obliquely propagating waves is subject to debates (Gary and Smith, 2009) Podesta et al., 2010). According to interplanetary measurements, these constituent waves may be kinetic Alfvén waves (Bale et al., 2005, Sahraoui et al., 2010) or fast-whistler waves (Gary and Smith, 2009). In a warm plasma such as the interplanetary solar wind, oblique Alfvén waves can generate a fast proton beam through Landau resonance (Li et al., 2010, Osmane et al., 2010). In a cold proton/electron plasma, quasi-perpendicular fast-whistler waves break up into Bernstein modes near the first few harmonics of proton cyclotron frequency (Li and Habbal, 2001 Markovskii et al., 2010). Due to the existence of minor ions in a solar coronalike plasma, oblique fast-whistler waves are linearly coupled with hybrid cyclotron waves (Li and Habbal, 2001). Depending on the abundance and relative speed with respect to protons, minor ions may change wave dispersion relation dramatically, induce a transition of wave polarization from right- to lefthanded sense, and can absorb more energy from oblique fast-cyclotron waves than Alfvén-cyclotron waves for intermediate angles $30^{\circ}-50^{\circ}$ of wave propagation (Li and Habbal, 2001). However, Alfvén-cyclotron and fast-cyclotron waves in the inner corona have few empirical constraints for their exact properties such as generation mechanisms, propagation modes, and power levels. With favorable plasma parameters, both Alfvén and fast waves can be potential candidates for preferential energization of minor ions in the solar wind. On the basis of current understanding, both fast-whistler and Alfvén-cyclotron waves at both quasi-parallel and quasi-perpendicular propagation angles probably contribute to kinetic-scale turbulence in the solar wind (Gary and Smith, 2009, He et al., 2011).

In this paper, both Alfvén and fast waves are investigated to explore their roles in energizing the low-beta multi-ion solar wind plasma in the inner corona. As a continuation to the work of Li and Habbal (2001), the linear mode conversion between fast-whistler and hybrid cyclotron waves is further quantified by a detailed parametric study of various plasma parameters. A quasi-linear theory of resonant wave-particle interaction (Marsch and Tu, 2001) is used, and we are able to study the parallel and perpendicular heating of solar wind species due to Landau and cyclotron resonances. We give our model in Section 2 and describe wave dispersion relations and accompanying electromagnetic field oscillations in Section 3 Then the roles of wave propagation angle, minor ion presence, relative flow of minor ions, and plasma temperature are successively analyzed in Sections 45, 6 and 7 . We discuss the ensuing nonlinear stage of wave-particle interaction in Section 8, and summarize our results in Section 9 .

\section{Methods}

To evaluate the energization effect of kinetic wave-particle interaction on the multi-species solar wind, two methods are sequentially combined in this pa- 
per. First, using linear Vlasov theory (Stix, 1992 Li and Habbal, 2001), the properties of Alfvén and fast waves such as their dispersion relations and electromagnetic polarizations are studied. Second, using a quasi-linear theory of resonant wave-particle interaction (Marsch and Tu, 2001), the previously derived wave properties are adopted to calculate the acceleration and heating rates of different plasma species. With the two-step approach, the wave-particle interaction can be quantified to demonstrate the most viable and efficient way for the solar wind energization.

Since we are interested in the main solar wind acceleration region, typical plasma conditions in the inner corona are chosen in our analyses. Given a background magnetic field $\mathbf{B}_{\mathbf{0}}$, a wave vector $\mathbf{k}$ is decomposed into a parallel component $k_{\|}$and a perpendicular one $k_{\perp}$. The propagation angle of a wave $\theta=\arctan \left(k_{\perp} / k_{\|}\right)$is defined with respect to $\mathbf{B}_{\mathbf{0}}$. Our Cartesian system of $(x, y, z)$ coordinates is constructed with $\mathbf{B}_{\mathbf{0}}$ along the $z$-axis and $\mathbf{k}$ within the $x z$-plane. The symbols and corresponding denotations used in this paper are summarized in Table 1 In this paper, only electrons e, protons p, and alpha particles $\alpha$ are considered in the solar wind. For simplicity, VDF of each species is approximated to be bi-Maxwellian. The parallel (perpendicular) thermal speed $v_{\| j}\left(v_{\perp j}\right)$ and gyrotropic $\operatorname{VDF} f_{j}\left(v_{\|}, v_{\perp}\right)$ are expressed as follows:

$$
\begin{aligned}
v_{\| j} & =\sqrt{2 k_{\mathrm{B}} T_{\| j} / m_{j}}, \\
v_{\perp j} & =\sqrt{2 k_{\mathrm{B}} T_{\perp j} / m_{j}}, \\
f_{j}(\mathbf{v}) & =f_{j}\left(v_{\|}, v_{\perp}\right)=\frac{1}{\pi^{3 / 2} v_{\| j} v_{\perp j}^{2}} \exp \left(-\frac{\left(v_{\|}-U_{\| j}\right)^{2}}{v_{\| j}^{2}}-\frac{v_{\perp}^{2}}{v_{\perp j}^{2}}\right) .
\end{aligned}
$$

The dispersion relation $\omega=\omega(\mathbf{k})$ and its inherent electromagnetic field $(\delta \mathbf{E}$, $\delta \mathbf{B})$ of a plasma wave are calculated using linear Vlasov theory (Stix, 1992, Li and Habbal, 2001). The complex wave frequency $\omega(\mathbf{k})=\omega_{\mathrm{r}}(\mathbf{k})+i \omega_{\mathrm{i}}(\mathbf{k})$, $\delta \mathbf{B}(\mathbf{k})$, and $\delta \mathbf{E}(\mathbf{k})$ are found as a function of wave vector $\mathbf{k}$. The damping rate $\left|\omega_{\mathrm{i}}\right|$ generally increases with wave number $k$. Gary (1993) suggested that heavily attenuated waves are described by a rough approximation:

$$
\omega_{\mathrm{i}}(\mathbf{k})<-\frac{\omega_{\mathrm{r}}(\mathbf{k})}{2 \pi}
$$

and that only weakly damped waves can persist. Given $\delta \mathbf{E}(\mathbf{k})$, polarization $P$ and unimodular electric vector $\mathbf{e}_{E}(\mathbf{k})$ are given by

$$
\begin{aligned}
P & =i \frac{\delta \mathbf{E}_{x}}{\delta \mathbf{E}_{y}} \frac{\omega_{\mathrm{r}}}{\left|\omega_{\mathrm{r}}\right|}, \\
\mathbf{e}_{E}(\mathbf{k}) & =\frac{\delta \mathbf{E}(\mathbf{k})}{|\delta \mathbf{E}(\mathbf{k})|} .
\end{aligned}
$$


Consequently, the magnetic field fluctuation $\delta \mathbf{B}(\mathbf{k})$ and its power spectrum $|\delta \mathbf{B}(\mathbf{k})|^{2}$ are

$$
\begin{aligned}
\delta \mathbf{B}(\mathbf{k}) & =\frac{1}{\omega(\mathbf{k})} \mathbf{k} \times \delta \mathbf{E}(\mathbf{k}) \\
|\delta \mathbf{B}(\mathbf{k})|^{2} & =\left(\frac{k}{\omega_{\mathrm{r}}(\mathbf{k})}\right)^{2}\left(1-\left|\mathbf{e}_{k} \cdot \mathbf{e}_{E}(\mathbf{k})\right|^{2}\right)|\delta \mathbf{E}(\mathbf{k})|^{2} \\
\mathbf{e}_{k} & =\frac{\mathbf{k}}{|\mathbf{k}|}=\frac{\mathbf{k}}{k}
\end{aligned}
$$

The wave-particle interaction via Landau and cyclotron resonances is efficient in transferring wave energy to particles in a collisionless plasma. With the introduction of Doppler-shifted frequency $\omega^{\prime}(\mathbf{k})=\omega_{\mathrm{r}}(\mathbf{k})-k_{\|} U_{\| j}$, the $n$ th-order resonant parallel speed in the bulk frame of species $j$ is defined as:

$$
w_{j}(\mathbf{k}, n)=\frac{\omega^{\prime}(\mathbf{k})-n \Omega_{j}}{k_{\|}}=\frac{\omega_{r}(\mathbf{k})-k_{\|} U_{\| j}-n \Omega_{j}}{k_{\|}} .
$$

Then, the resonant factor $\xi_{j}$ for species $j$ is defined as:

$$
\xi_{j}(\mathbf{k}, n)=\frac{w_{j}(\mathbf{k}, n)}{v_{\| j}}=\frac{\omega_{r}(\mathbf{k})-k_{\|} U_{j}-n \Omega_{j}}{k_{\|} v_{\| j}}, \quad n=0, \pm 1, \pm 2, \ldots .
$$

Here $n=0$ corresponds to the Landau resonance, $n \neq 0$ the cyclotron resonance. Only the Landau resonance $\xi^{0}$ and the first-order cyclotron resonance $\xi^{+}$may appear for the plasma parameters prescribed in Tables 2 and 3 .

The acceleration and heating rates of a species due to any wave mode were formulated by Marsch and Tu (2001) using a quasi-linear theory, and are applied in this paper. In the quasi-linear theory, different waves are simply considered to be linearly superposed without any interference. Specifically, for species $j$, its parallel acceleration rate $a_{\| j}$, parallel heating rate $Q_{\| j}$, and perpendicular heating rate $Q_{\perp j}$ are integrated over various wave modes $M$ and all resonance orders $n$, and are listed below:

$$
\begin{gathered}
\left(\begin{array}{c}
\frac{\partial}{\partial t} U_{\| j} \\
\frac{\partial}{\partial t} v_{\| j}^{2} \\
\frac{\partial}{\partial t} v_{\perp j}^{2}
\end{array}\right)=\sum_{M} \int_{-\infty}^{+\infty} d^{3} k \frac{|\delta \mathbf{B}(\mathbf{k})|^{2}}{8 \pi}\left(\begin{array}{c}
a_{\| j} / m_{j} \\
Q_{\| j} / m_{j} \\
Q_{\perp j} / m_{j}
\end{array}\right) \\
\left(\begin{array}{c}
a_{\| j} / m_{j} \\
Q_{\| j} / m_{j} \\
Q_{\perp j} / m_{j}
\end{array}\right)=\sum_{n=-\infty}^{+\infty}\left(\frac{1}{2 \pi}\right)^{3}\left(\frac{\Omega_{j}}{k}\right)^{2} \frac{1}{1-\left|\mathbf{e}_{\mathbf{k}} \cdot \mathbf{e}_{E}(\mathbf{k})\right|^{2}} R_{j}(\mathbf{k}, n)\left(\begin{array}{c}
k_{\|} \\
2 k_{\|} w_{j}(\mathbf{k}, n) \\
n \Omega_{j}
\end{array}\right) .
\end{gathered}
$$

Herein, the resonance function $R_{j}(\mathbf{k}, n)$ gives energy absorption of species $j$ from a wave mode $|\delta \mathbf{B}(\mathbf{k})|^{2}$. An electric field oscillation could be factorized into a left-handed polarization and a right-handed one. The factorized components 
of unimodular electric field $\mathbf{e}_{E}(\mathbf{k})$ and total electric field $\delta \mathbf{E}(\mathbf{k})$ are expressed as

$$
\begin{aligned}
\mathbf{e}_{E}^{ \pm}(\mathbf{k}) & =\mathbf{e}_{E x}(\mathbf{k}) \pm i \mathbf{e}_{E y}(\mathbf{k}) \\
\delta \mathbf{E}^{ \pm}(\mathbf{k}) & =\delta \mathbf{E}_{x}(\mathbf{k}) \pm i \delta \mathbf{E}_{y}(\mathbf{k}) .
\end{aligned}
$$

In Equations (20) and (21), subscripts + and - denote left- and right-handed polarizations, respectively. The transverse wave number $k_{\perp}$ is compared with the ion cyclotron radius of thermal motions $v_{\perp j} / \Omega_{j}$, and hence described by a parameter $\lambda_{j}$.

$$
\lambda_{j}=\frac{1}{2}\left(\frac{k_{\perp} v_{\perp j}}{\Omega_{j}}\right)^{2} .
$$

Further, the resonance function $R_{j}(\mathbf{k}, n)$ is defined as

$$
\begin{aligned}
& R_{j}(\mathbf{k}, n)=2 N_{j}(\mathbf{k}, n) \exp \left(-\lambda_{j}\right)\left[a_{-} I_{n-1}\left(\lambda_{j}\right)+a_{+} I_{n+1}\left(\lambda_{j}\right)+a_{0} I_{n}\left(\lambda_{j}\right)\right], \\
& N_{j}(\mathbf{k}, n)=\sqrt{\pi} \frac{k_{\|}}{\left|k_{\|}\right|} \exp \left[-\xi_{j}^{2}(\mathbf{k}, n)\right] \times\left\{\xi_{j}(\mathbf{k}, n) \frac{T_{\perp j}}{T_{j \|}}+n \frac{\Omega_{j}}{k_{\|} v_{\| j}}\right\}, \\
& a_{0}(\mathbf{k}, n)=2\left(\frac{\omega(\mathbf{k})-n \Omega_{j}}{k_{\|} v_{\perp j}}\right)^{2}\left|\mathbf{e}_{E z}\right|^{2}-2 \operatorname{Im}\left\{\mathbf{e}_{E y}^{*} \mathbf{e}_{E z}\right\} \frac{k_{\perp}}{k_{\|}} \frac{\omega(\mathbf{k})-n \Omega_{j}}{\Omega_{j}}+2 \lambda_{j}\left|\mathbf{e}_{E y}\right|^{2},
\end{aligned}
$$

$a_{\mp}(\mathbf{k}, n)= \pm \frac{n}{2}\left|\mathbf{e}_{E}^{ \pm}\right|^{2} \pm \operatorname{Re}\left\{\mathbf{e}_{E z}^{*} \mathbf{e}_{E}^{ \pm}\right\} \frac{k_{\perp}}{k_{\|}} \frac{\omega(\mathbf{k})-n \Omega_{j}}{\Omega_{j}} \mp \lambda_{j} \operatorname{Im}\left\{\mathbf{e}_{E y}^{*} \mathbf{e}_{E}^{ \pm}\right\}$

Here $I$ is a modified Bessel function. For parallel propagation, the above form of $R_{j}(\mathbf{k}, n)$ can be largely simplified as

$$
R_{j}\left(0, k_{\|}, n\right)=N_{j}\left(0, k_{\|}, n\right)\left[\delta_{n, 1}\left|\mathbf{e}_{E}^{+}\right|^{2}+\delta_{n,-1}\left|\mathbf{e}_{E}^{-}\right|^{2}+4 \delta_{n, 0}\left|\mathbf{e}_{E z}\right|^{2}\left(\frac{\omega\left(k_{\|}\right)}{k_{\|} v_{\perp j}}\right)^{2}\right] .
$$

Note that the resonance function $R_{j}(\mathbf{k}, n)$ essentially depends on the resonance factor $\xi_{j}$ and the square of the re-scaled perpendicular wave number $\lambda_{j}=$ $\left(k_{\perp} v_{\mathrm{A}} / \Omega_{j}\right)^{2} / 2$ as exponential functions of $\exp \left(-\xi_{j}^{2}\right)$ in $N_{j}(\mathbf{k}, n)$ (Equation (24)) and $\exp \left(-\lambda_{j}\right)$ in $R_{j}(\mathbf{k}, n)$ (Equation (23) ) respectively. As $\xi_{j}$ or $\lambda_{j}$ is increased, $\exp \left(-\xi_{j}^{2}\right)$ or $\exp \left(-\lambda_{j}\right)$ dramatically declines to zero, and energization effect quickly becomes negligible.

We assume that plasma turbulence $|\delta \mathbf{B}(\mathbf{k})|^{2}$ is the ultimate energy source of the solar wind. For simplicity, energization contributions of Alfvén and fast waves are individually evaluated in terms of the energization rates $a_{\|}, Q_{\|}$, and $Q_{\perp}$ in Equation (19). Both Alfvén and fast waves are assumed to have the same PSD $|\delta \mathbf{B}(\mathbf{k})|^{2}$ at the same wave vector $\mathbf{k}$. A specific form for $|\delta \mathbf{B}(\mathbf{k})|^{2}$ in Equation (18) is beyond the scope of this paper. Initial prescriptions of various plasma parameters are given in Tables 2 and 3 . Then the values of $a_{\|}, Q_{\|}$, and $Q_{\perp}$ (Equation (19)) normalized to $|\delta \mathbf{B}(\mathbf{k})|^{2}$ (Equation (18)) are derived by a combined two-step approach (Li and Habbal, 2001; Marsch and Tu, 2001). Here a detailed parametric study is conducted to analyze how cascaded turbulence energy is repartitioned among multiple constituent plasma species. 


\section{Dispersion Relation and Electromagnetic Field Oscillation}

Dispersions of Alfvén and fast waves in a proton/alpha/electron plasma are illustrated for various propagation angles $\theta$ in Figures 1, 2, and 3, In the presence of alpha particles, an Alfvén-cyclotron wave branch of $0<\omega_{\mathrm{r}}<\Omega_{\mathrm{p}}$ is generally split into two parts near the cyclotron frequency of alpha particles $\Omega_{\alpha}$. The lower branch of $0<\omega_{\mathrm{r}}<\Omega_{\alpha}$ corresponds to Alfvénic alpha cyclotron waves, and the upper one of $\Omega_{\alpha}<\omega_{\mathrm{r}}<\Omega_{\mathrm{p}}$ refers to hybrid alpha-proton cyclotron waves. Obviously, the alpha cyclotron resonances occur at much smaller wave numbers for the hybrid cyclotron waves than the Alfvén-cyclotron waves. Though having not been measured experimentally, kinetic hybrid waves with $\omega_{\mathrm{r}} \approx \Omega_{\alpha}$ and $k \approx 0$ may be locally generated in a cold multi-ion plasma as a result of linear mode coupling with fast-whistler waves (Li and Habbal, 2001). Avoiding a crossing between the $\omega-k$ dispersion curves of hybrid ion-ion cyclotron and fast-whistler waves, fast-whistler waves are possibly transformed into fast-cyclotron waves. By comparing Figure 13 with 1r, one can see that the dispersion of oblique fast waves is dramatically changed by the presence of alpha particles in a dominant electron/proton plasma. Fast-whistler and alpha-proton cyclotron waves exchange their identities at alpha cyclotron frequency $\omega_{\mathrm{r}}=\Omega_{\alpha}=0.5 \Omega_{\mathrm{p}}$. Such linear mode conversion in Figure 1. occurs when fast waves are oblique enough, i.e. the propagation angle $\theta \geq 31^{\circ}$ in this paper (Figure 3 a). Once mode conversion happens, the conversion point in the dispersion curve is basically at one identical point of $\omega_{\mathrm{r}} / \Omega_{\mathrm{p}}=0.5$ and $k v_{\mathrm{A}} / \Omega_{\mathrm{p}}=0.5$, irrespective of wave direction $\theta$. At a sufficiently long wavelength, fluctuations of both Alfvén and fast waves are essentially undamped. For ion cyclotron waves, as the parallel wave number $k_{\|}$reaches a characteristic dissipation value $k_{\mathrm{d}}$, relevant ions are in cyclotron resonance and the damping begins abruptly. In a proton/electron plasma of $0.001 \leq \beta_{\mathrm{p}} \leq 0.1$, $k_{\mathrm{d}} \approx \Omega_{\mathrm{p}} / v_{\mathrm{A}}$ for the proton cyclotron resonance was given by Gary and Borovsky (2004); In a cold alpha/proton/electron plasma, $k_{\mathrm{d}} \approx \Omega_{\alpha} / v_{\mathrm{A}}=0.5 \Omega_{\mathrm{p}} / v_{\mathrm{A}}$ for the alpha cyclotron resonance is found in this paper. Specifically, for nearly parallel Alfvén waves of $\theta=1^{\circ}$, the damping rate $\left|\omega_{\mathrm{i}} / \Omega_{\mathrm{p}}\right|$ is increased from $2 \times 10^{-7}$ at $k v_{\mathrm{A}} / \Omega_{\mathrm{p}}=0.45$ to $4 \times 10^{-3}$ at $k v_{\mathrm{A}} / \Omega_{\mathrm{p}}=0.6$ (Figure 1). Meanwhile, damping of fast-whistler waves is only pronounced at harmonic ion cyclotron frequencies for oblique propagation ( $\mathrm{Li}$ and Habbal, 2001). However, due to linear mode conversion, fast-cyclotron waves will experience a dramatic increase of damping rate $\left|\omega_{\mathrm{i}}\right|$, as shown in Figure 11. Heavily damped waves disappear swiftly, as described in Equation (10) (Gary, 1993). Approaching ion cyclotron resonance frequency, smaller parallel wave numbers $k_{\|}$and larger total wave numbers $k$ are found as $\theta$ increases to be more oblique, especially at $\theta \approx 90^{\circ}$ (Figures 2 and 3). When a turbulence cascade is present, Alfvén-cyclotron, fastwhistler, and fast-cyclotron waves may continuously transfer wave energy from very low frequencies along their respective dispersion curves, and ultimately become constituent modes of kinetic turbulence at ion cyclotron frequencies.

Electromagnetic behaviors of Alfvén and fast waves are displayed in Figures 44 5. and 6. As fast-cyclotron and Alfvén-cyclotron waves become more oblique, phase speeds $\omega_{\mathrm{r}} / k$ involving cyclotron resonances are gradually reduced towards 0 , and electric field oscillations $\delta \mathbf{E}$ become more aligned with wave vector $\mathbf{k}$. 
The values of $\omega_{\mathrm{r}} / k$ and $\left(1-\left|\mathbf{e}_{k} \cdot \mathbf{e}_{E}\right|\right)$ determine the ratio of electric to magnetic field fluctuations $|\delta \mathbf{E}|^{2} /|\delta \mathbf{B}|^{2}$ (Equation (14)). For Alfvén-cyclotron waves with $0^{\circ} \leq \theta<90^{\circ}$ (Figures $4 \mathrm{a}$ and $5 \mathrm{a}$ ) and fast-cyclotron waves with $31^{\circ} \leq \theta<90^{\circ}$ (Figures 4a and $6 \mathrm{a}$ ),$|\delta \mathbf{E}|^{2} /|\delta \mathbf{B}|^{2}$ increases when $\theta$ becomes larger. At oblique enough angles, both fast and Alfvén waves are nearly electrostatic and linearly polarized as a result of $\delta \mathbf{E}$ being almost parallel to $\mathbf{k}$. In particular, nearlyperpendicular fast waves become almost electrostatic, as $|\delta \mathbf{E}|^{2} /\left(|\delta \mathbf{B}|^{2} v_{\mathrm{A}}^{2}\right)$ could be as high as 50 at $k v_{\mathrm{A}} / \Omega_{\mathrm{p}}=3$ (Figures 4 and 6 a). As a result, electric field fluctuations $|\delta \mathbf{E}|$ of fast waves are significantly enhanced over their corresponding magnetic field fluctuations $|\delta \mathbf{B}|$. As for Alfvén waves, $|\delta \mathbf{E}|$ and $|\delta \mathbf{B}| \cdot v_{\mathrm{A}}$ are equally strong for quasi-perpendicular propagation (Figures 4 e and 5 a), though $|\delta \mathbf{E}|$ is far less than $|\delta \mathbf{B}| \cdot v_{\mathrm{A}}$ at large $k$ for parallel propagation (Figures 4 . and 5 a). Meanwhile, for oblique propagation, parallel electric field fluctuations $\left|\delta E_{\|}\right|$are enhanced over the whole spectrum. The overall sense of the electric field polarization is left-handed for parallel Alfvén waves (Figures 4 and 5b), right-handed for parallel fast waves (Figure 6 b), and nearly linearly polarized for perpendicular Alfvén and fast waves (Figures 40, 4f, 5b, and 6b). Obviously, linear mode conversion from fast waves to alpha cyclotron waves near alpha cyclotron frequency $\Omega_{\alpha}$ abruptly increases the left-handed electric field oscillation (Figure 6b). When present in the inner corona, both Alfvén and fast waves could heat plasma species through Landau and cyclotron resonances. If a resonant factor $\left|\xi_{j}\right|$ is large, only particles in the remote tail of the VDF of species $j$ are in resonance with the wave. In linear Vlasov theory, a resonance factor of $\left|\xi_{j}\right| \leq 3$ is a necessary but not a sufficient condition for non-negligible wave-particle interaction (Gary and Borovsky, 2004). Both $\left|\xi_{j}^{0}\right| \leq 3$ and parallel electric field oscillation $\left|\delta E_{\|}\right|>0$ are required for noticeable Landau resonance; Similarly, both $\left|\xi_{j}^{+}\right| \leq 3$ and left-handed electric field oscillation $\left|\delta E^{+}\right|>0$ correspond to cyclotron resonance. Due to nearly zero $\left|\delta E_{\|}\right|$accompanying quasiparallel waves, Landau resonances are very weak even with the synchronous condition of wave and particle phases, i.e. $\left|\xi_{j}^{0}\right| \leq 3$. Conspicuous streaks of $\xi_{\mathrm{p}}^{+}$ from $0^{\circ}<\theta<31^{\circ}$ (Figure 6e) and $\xi_{\alpha}^{+}$from $0^{\circ}<\theta<90^{\circ}$ (Figure 6f) correspond to locally enhanced damping of fast waves at ion cyclotron frequencies $\Omega_{\alpha}$ and $\Omega_{\mathrm{p}}$. However, as explained by Li and Habbal (2001), the localized cyclotron damping of fast waves by species $j$ is unlikely to be important because of its localized occurrence in a very narrow frequency band across $\Omega_{j}$. Through Landau and/or cyclotron resonances, different plasma species absorbs energy from the turbulence power spectrum $|\delta \mathbf{B}(\mathbf{k})|^{2}$ accordingly.

\section{Effect of Wave Propagation Angle}

The energization of any plasma species $j$ due to turbulence $\operatorname{PSD}|\delta \mathbf{B}(\mathbf{k})|^{2}$ is estimated by quasi-linear transport coefficients $\left(a_{\| j}, Q_{\| j}\right.$, and $\left.Q_{\perp j}\right)$ in Equation (19) and visualized in Figures 7 and 8 . Alfvén and fast waves at each $\mathbf{k}$ are assumed to be equally strong; the magnitude of fluctuation $|\delta \mathbf{B}|$ is the same for both waves. The parallel acceleration $a_{\| j}$, parallel heating $Q_{\| j}$, and perpendicular heating $Q_{\perp j}$ are basically determined by the resonance function $R$ 
in Equation (23). Different species show different behaviors in terms of their energization from wave damping. Alfvén-cyclotron waves essentially do not interact with protons, because their asymptotic frequency $\Omega_{\alpha}$ is far away from the proton cyclotron frequency $\Omega_{\mathrm{p}}$ (Figure $2 \mathrm{a}$ ). Fast-whistler waves with propagation angles $0^{\circ} \leq \theta<31^{\circ}$ are essentially undamped, as shown in Figure 8, Noticeable energy recipients of Alfvén waves are alpha particles when $0^{\circ} \leq \theta<80^{\circ}$ and electrons when $80^{\circ} \leq \theta<90^{\circ}$ (Figure 7); those of fast waves are alpha particles with $31^{\circ} \leq \theta<90^{\circ}$, protons with $31^{\circ} \leq \theta<80^{\circ}$, and electrons with $80^{\circ} \leq \theta<90^{\circ}$ (Figure 8). The alpha cyclotron waves in Figure 7 have similar energization effects as the proton cyclotron waves in Figure 8 , though being damped more heavily. The strongest energy recipient among all plasma species is the alpha particles via alpha cyclotron resonances with fast-cyclotron waves when $31^{\circ} \leq \theta<90^{\circ}$ (Figures $8 \mathrm{a}$-c). Such an alpha energization from fast-cyclotron waves is increasingly significant, as the waves become more oblique towards $\theta=90^{\circ}$. Moreover, for effective energization from fast-cyclotron waves, alpha particles occupy a lower part of the $k_{\|}-\theta$ parameter space than either protons or electrons. Based on the turbulence cascade scenario, the PSD is absorbed by resonant alpha particles from fast-cyclotron waves at lower frequencies and wave numbers, and hence has larger fluctuation amplitudes $|\delta \mathbf{B}|^{2}$. As a result, the combination of the largest $|\delta \mathbf{B}|^{2}$ in Equation (18) and the largest $\left(a_{\| \alpha}, Q_{\| \alpha}\right.$, $Q_{\perp \alpha}$ ) in Equation (19) are responsible for the strongest alpha energization. In addition, compared with alpha cyclotron frequency $\Omega_{\alpha}$, frequencies $\omega_{\mathrm{r}}$ at the alpha cyclotron resonance are slightly higher for fast-cyclotron waves (Figure 1r) and slightly lower for Alfvén-cyclotron waves (Figures 1a and Tr). Consequently, the first-order parallel resonant speeds $w_{\alpha}(\mathbf{k}, 1)$ are positive for fast-cyclotron waves and negative for Alfvén-cyclotron waves (Equation (16)). According to Equations (18) and (19), fast-cyclotron and Alfvén-cyclotron waves are responsible for parallel heating and cooling of alpha particles respectively. With Alfvén waves, it is much easier for the VDF of alpha particles to develop into a kinetic anisotropy of $T_{\perp \alpha}>T_{\| \alpha}$, and then trigger an ion cyclotron instability of alpha particles. In a cold plasma of $\beta_{\mathrm{p}}=0.01$, alpha particles are the primary energy recipient from the damping of both Alfvén and fast waves. This preferential energization of alpha particles is much more significant by oblique fast-cyclotron waves via linear mode conversion from their corresponding fast-whistler waves.

\section{Influence of Minor Ion Presence}

Without alpha particles in a proton/electron plasma, oblique fast-whistler waves can still be linearly converted to Bernstein waves at harmonic proton cyclotron frequencies of $\Omega_{\mathrm{p}}$ and $2 \Omega_{\mathrm{p}}$ (Li and Habbal, 2001; Markovskii et al., 2010). At a perpendicular angle of $\theta=90^{\circ}$, Bernstein waves are purely electrostatic, do not damp, and cannot affect ions (Stix, 1992). When $\theta$ deviates from $90^{\circ}$, Bernstein waves are strongly damped. With linear mode coupling, highly oblique fast-Bernstein waves are strongly dissipated by protons, as previously demonstrated from linear Vlasov analyses (Li and Habbal, 2001) and hybrid simulation (Markovskii et al., 2010) of fast-wave cascade spectrum. With the absence of 
alpha particles, energization rates of protons and electrons are estimated by Equation (19). Only differing in the abundance of alpha particles $n_{\alpha}=0.02 n_{\mathrm{p}}$ (Tables 2] and 3), the two sets of initial plasma parameters in Sections 4 and 5 result in dramatically different energizations. Linear mode conversion from fast to Bernstein waves occurs, when the fast waves are oblique enough, i.e., propagation angles $\theta \geq 64^{\circ}$. Specifically, such a conversion point of fast-Bernstein wave branch is at $2 \Omega_{\mathrm{p}}$ for $64^{\circ} \leq \theta<87^{\circ}$ and $\Omega_{\mathrm{p}}$ for $87^{\circ} \leq \theta<90^{\circ}$. Energization of protons and electrons are noticeable for $64^{\circ} \leq \theta<85^{\circ}$ and $80^{\circ} \leq \theta<90^{\circ}$, respectively. Because of the linear mode coupling, fast-whistler waves are intercepted at frequencies $\omega_{\mathrm{i}} \approx \Omega_{\alpha}$ for alpha cyclotron waves and $\omega_{\mathrm{i}} \approx \Omega_{\mathrm{p}}$ (or $2 \Omega_{\mathrm{p}}$ ) for Bernstein waves. Parallel electric field $\left|\delta E_{\|}\right|$between $\Omega_{\alpha}$ and $\Omega_{\mathrm{p}}$ of fast-Bernstein waves are responsible for a much stronger electron energization in contrast with fast-cyclotron waves. Protons undergo larger energization by fast-Bernstein waves than by fast-cyclotron waves, particularly in perpendicular heating $Q_{\perp \mathrm{p}}$. However, fast-cyclotron waves primarily energize alpha particles, and such a strong ion energization cannot be found in fast-Bernstein waves. When alpha particles are present, fast waves are coupled to cyclotron waves instead of Bernstein waves as a result of the turbulence cascade. Approaching alpha cyclotron frequency $\Omega_{\alpha}$ (instead of $\Omega_{\mathrm{p}}$ ), a fast-cyclotron wave has a smaller frequency $\omega_{\mathrm{r}}$, a smaller wave number $k$, and larger wave energy density $|\delta \mathbf{B}(\mathbf{k})|^{2}$ based on the turbulence cascade scenario. Moreover, the angle threshold for linear mode coupling is low for fast-cyclotron waves $\left(\theta \geq 31^{\circ}\right)$ and high for fastBernstein waves $\left(\theta \geq 64^{\circ}\right)$. Therefore, the existence of alpha particles could lead to more efficient damping of fast waves.

\section{Role of Relative Flow of Minor Ions}

Wave-particle interaction in a multi-ion plasma may be significantly modulated by a differential speed $U_{\| j}$ of minor ion $j$. Here $U_{\| j}$ is defined as the bulk flow speed relative to protons. The dependence of energization on $U_{\| \alpha}$ in a proton/alpha/electron plasma is presented in Figure 9 for Alfvén waves and Figure 10 for fast waves along propagation angle $\theta=60^{\circ}$. Initially cold alpha particles are swiftly accelerated and heated by both Alfvén-cyclotron and fast-cyclotron waves. Fast-cyclotron waves resonate with alpha particles at frequencies $\omega_{\mathrm{i}} \approx \Omega_{\alpha}$ and protons at $\omega_{\mathrm{i}} \approx \Omega_{\mathrm{p}}$, and energize alpha particles at a low wave number $k$ (Figures 10a-c) and protons at a high $k$ (Figures 10 $\mathrm{d}-\mathrm{f}$ ). Obviously, alpha particles are energized much more strongly than protons for Alfvén-cyclotron waves at $U_{\| \alpha}<0.2 v_{\mathrm{A}}$ (Figure 91) and for fast-cyclotron waves at $U_{\| \alpha}<0.6 v_{\mathrm{A}}$ (Figure 100). When $U_{\| \alpha}$ reaches $0.2 v_{\mathrm{A}}$, Alfvén-cyclotron waves at $\theta=60^{\circ}$ begin to resonate with protons, and alpha particles lose the initial energization advantage. Meanwhile, oblique fast-cyclotron waves continue to preferentially energize alpha particles. The appearance of fast-cyclotron waves occurs for $\theta \geq 31^{\circ}$ at $U_{\| \alpha} / v_{\mathrm{A}}=0$ (Figure 8) and $\theta>60^{\circ}$ at $U_{\| \alpha} / v_{\mathrm{A}}=0.6$ (Figure 10). Obviously, as a response to continuously increasing $U_{\| \alpha}$, the minimum threshold of wave propagation angle $\theta$ at which the coupling between fast-whistler and hybrid alpha-proton cyclotron waves occurs is increased accordingly. As a result of 
continuous preferential energization, alpha particles gradually accumulate high enough $U_{\| \alpha}$ and temperature anisotropy $T_{\perp_{\alpha}} / T_{\| \alpha}$. When alpha particles lose resonance, the major wave-particle interaction is proton cyclotron resonance for Alfvén-cyclotron waves (Figures 9-f-f) and electron Landau resonance for fastwhistler waves (Figure $10 \mathrm{~h}$ ). Productions of much higher $U_{\| \alpha}$ and $T_{\perp \alpha} / T_{\| \alpha}$ are inhibited, as excessive free energy of alpha beam and kinetic anisotropy in the VDF of alpha particles would be released to excite Alfvén-cyclotron waves (Figures $9 \mathrm{a}-\mathrm{c}$ ). The self-consistent evolution of alpha particles in the parametric space of $U_{\| \alpha}$ and $T_{\perp \alpha} / T_{\| \alpha}$ could be further investigated by particle simulations. Here from Vlasov analyses of various plasma parameters, both fast and Alfvén waves are likely to contribute to the eventually sustainable differential speed and temperature anisotropy of minor ions in the solar wind.

\section{Dependence on Plasma Temperature}

Fast-cyclotron waves only exist in a cold plasma, because linear coupling of fast-whistler and hybrid-cyclotron waves disappears for large plasma beta. Parametric studies of proton beta $\beta_{\mathrm{p}}$ are performed for Alfvén and fast waves along propagation angle $\theta=60^{\circ}$ (Tables 2 and 3). Generally, in an increasingly hot plasma, waves are increasingly damped, and their effective $\omega_{\mathrm{r}}-k$ dispersion curves are consequently shortened according to Equation (10). Max. $\left(k_{\|}\right)$ generally decreases with an increasing $\beta_{\mathrm{p}}$, which is estimated at each $\beta_{\mathrm{p}}$ in the $k_{\|}-\beta_{\mathrm{p}}$ parameter domain according to weak damping condition (Equation (10) $)$. Exceptional increases of Max. $\left(k_{\|}\right)$are found from a transition of $-1.2<\log _{10}\left(\beta_{\mathrm{p}}\right)<-1$ and a jump across $\log _{10}\left(\beta_{\mathrm{p}}\right)=-0.95$. Such exceptions in the trend of $\operatorname{Max} .\left(k_{\|}\right)$versus $\beta_{\mathrm{p}}$ are due to a significant dispersion change of corresponding wave branch. As $\beta_{\mathrm{p}}$ increases, the dispersion of Alfvén waves finally changes to cross the alpha cyclotron frequency $\Omega_{\alpha}$, and fast waves ultimately cease the conversion to hybrid alpha-proton waves. The sudden change of wave dispersion corresponds to a certain value of $\beta_{\mathrm{p}}$. The critical point is $\log _{10}\left(\beta_{\mathrm{p}}\right)=-1.2$ for Alfvén waves and $\log _{10}\left(\beta_{\mathrm{p}}\right)=-0.95$ for fast waves. Across the critical points, wave branches extend to higher frequencies $\omega_{\mathrm{r}}$, and survive for higher wave numbers $k_{\|}$. Alpha particles are the chief energy recipient from both Alfvén-cyclotron waves and fast-cyclotron waves $\left(\log _{10}\left(\beta_{\mathrm{p}}\right)<-0.95\right)$. The energization effects of Alfvén-cyclotron waves weaken as $\beta_{\mathrm{p}}$ increases, while those of fast-cyclotron waves peak in the range of $-2.2<\log _{10}\left(\beta_{\mathrm{p}}\right)<-1.2$. In addition, without mode conversion for $\log _{10}\left(\beta_{\mathrm{p}}\right) \geq-0.95$, oblique fast-whistler waves not only energize ions around ion cyclotron frequencies $\left(\Omega_{\alpha}\right.$ and $\left.\Omega_{\mathrm{p}}\right)$, but also dissipate a noticeable portion of wave energy to electrons in the form of parallel heating $Q_{\| \mathrm{e}}$. To the energy absorption of fast-whistler waves, the contribution of electrons is comparable to that of protons and alpha particles in a warm plasma. Only in a cold plasma, linear coupling of fast and cyclotron waves exists, and the electron heating could be negligible for damping of fast-cyclotron waves. Hence, in the inner corona with $\beta_{\mathrm{p}} \approx 0.01$, oblique fast-cyclotron waves are expected to play a more important role in energizing minor ions, provided that they are equally as strong as Alfvén-cyclotron waves. 


\section{Discussion on Nonlinear Stage of Wave-Particle Interaction}

It is still an enigma how the macro-scale heating and acceleration of the solar wind are powered by waves on ion kinetic scales and how these waves are generated by multi-scale coupling. The solar wind turbulence becomes fully developed on a time scale that is very short, compared to the expansion time of the solar wind along open magnetic flux tubes. So the turbulence evolution in the wave vector space could be considered to develop in a spatially homogeneous plasma, so we can adopt spatially uniform energization rates (Equation (19)) in this paper (see also Cranmer and Ballegooijen (2003)). In Equation (19), $a_{\| j}$ is probably not an important contributor to the solar wind acceleration, as the magnetic mirror force due to anisotropic heating of $Q_{\| j}$ and $Q_{\perp j}$ is typically stronger. A plasma species $j$ is energized by dissipation of its ion cyclotron waves. In a frame moving with the phase speed of the waves, a diffusion in the VDF of species $j$ (Equation (9) ) occurs along contours of constant energy. Such a diffusion process is faster for species $j$ with a lower charge-to-mass ratio. This wave-particle interaction was formulated into a kinetic shell model (Isenberg, 2001), which quantitatively describes ion energization by bidirectionally propagating ion cyclotron waves. As a result, the VDF in Equation (9) is gradually deviated from its initially Maxwellian distribution. The quasi-linear coefficients of Equation (18) are only valid in a small deviation from the Maxwellian VDF of particles (Equation (9) ) and a small damping rate of plasma waves (Equation (10)). Owing to these limitations, the analytical method in this paper can only describe the initial quasi-linear stage of wave-particle interaction. The ensuing nonlinear stage is beyond the scope of this paper, though it can be self-consistently described by full particle simulation.

\section{Conclusions and Summary}

Using the linear Vlasov wave theory and quasi-linear resonant wave-particle interaction for a low-beta multi-species plasma (Li and Habbal, 2001; Marsch and Tu, 2001) fast and Alfvén waves (and their dispersion relations and electromagnetic field fluctuations) are analyzed, and then applied to energize a proton/electron/alpha plasma with a low proton beta of $\beta_{\mathrm{p}} \approx 0.01$. In this paper, we assume that (1) low-frequency Alfvén and fast waves are launched from the solar surface with the same spectral shape and the same amplitude of PSD, (2) these macro-scale waves/turbulences continuously cascade towards kinetic scales at ion cyclotron frequencies and ion inertial scales, and (3) the transported kinetic-scale waves energize solar wind species through cyclotron and Landau resonances. If the wave propagation angle $\theta$ is oblique with respect to the background magnetic field, fast-whistler waves can be linearly coupled with hybrid alpha-proton cyclotron waves. Both fast-cyclotron and Alfvén-cyclotron waves are important for preferential heating and acceleration of minor ions. In this paper, noticeable energization of alpha particles requires oblique propagation for fast-cyclotron waves $\left(\theta \geq 31^{\circ}\right)$ and non-perpendicular propagation for Alfvén-cyclotron waves $\left(\theta<80^{\circ}\right)$. Nearly perpendicular Alfvén-cyclotron waves at $80^{\circ} \leq \theta<90^{\circ}$ significantly degenerate their capabilities of energizing minor ions due to very large 
perpendicular wave numbers at ion cyclotron frequencies. Only through complex nonlinear processes, nearly perpendicular Alfvén waves may heat ions. For instance, Cranmer and Ballegooijen (2003) proposed that the damping of kinetic Alfvén waves successively leads to electron beams, Langmuir turbulence, and Debye-scale electron phase space holes, and ions are perpendicularly heated via a collision-like scenario. Meanwhile, oblique fast-cyclotron waves have a larger lefthandedly polarized electric field $\left|\delta \mathbf{E}^{+}\right|$than the corresponding Alfvén-cyclotron waves, because the total electric field fluctuation $|\delta \mathbf{E}|$ of fast waves is stronger enough to over-compensate its smaller ratio of left- to right-handedly polarized electric fields $\left|\delta \mathbf{E}^{+}\right| /\left|\delta \mathbf{E}^{-}\right|$. Moreover, in contrast to Alfvén-cyclotron waves, alpha cyclotron resonance happens at a smaller wave number for fast-cyclotron waves especially at $\theta \approx 90^{\circ}$, where the turbulence PSD has a larger local amplitude. Hence, fast-cyclotron waves have a greater capability of energizing solar wind ions than Alfvén-cyclotron waves. Interacting with Alfvén-cyclotron and fast-cyclotron waves, alpha particles are preferentially energized as the primary recipient of wave energy. In contrast to alpha particles, electrons are generally less energized. When the differential speed $U_{\| \alpha}$ of alpha particles is high enough, Alfvén-cyclotron waves lose alpha cyclotron resonance much earlier than fast-cyclotron waves. For example, at $\theta=60^{\circ}$, alpha cyclotron resonance disappears at $U_{\| \alpha}=0.2 v_{\mathrm{A}}$ for Alfvén-cyclotron waves and $U_{\| \alpha}=0.6 v_{\mathrm{A}}$ for fastcyclotron waves. When alpha particles lose cyclotron resonance, fast-cyclotron waves restore to fast-whistler waves without linear mode conversion, and Alfvéncyclotron waves begin to mainly energize protons. Moreover, an extremely fast beam and/or a high temperature anisotropy of minor ions could release their excessive kinetic energy to excite ion cyclotron waves. Therefore both fast and Alfvén waves are likely to contribute to the eventually sustainable and balanced differential speed and temperature anisotropy of minor ions in the solar wind.

Linear mode conversion between fast-whistler and hybrid cyclotron waves due to the presence of minor ions could be an efficient mechanism to dissipate wave energy into minor ions. In a warm plasma in the interplanetary space, such mode coupling vanishes, and parallel electron heating by Landau damping of oblique fast-whistler waves becomes non-negligible. As demonstrated in this paper and the previous work of Li and Habbal (2001), fast-cyclotron waves besides Alfvéncyclotron waves could also be considered as a promising candidate for preferential heating and acceleration of minor ions in the inner corona. Since fast and Alfvén waves heat the solar wind ions in very different ways, it is important to know the exact nature of plasma turbulence at kinetic scales. In this regard, observation data such as Cluster archive can continue to address the issue.

Acknowledgements This research was supported by a grant from the Science \& Technology Facilities Council (STFC) to the Aberystwyth University, UK. We are very grateful to the anonymous referee for his/her thoughtful and constructive comments, which have greatly improved the quality of this paper. We also sincerely thank Prof. Takashi Sakurai and Mr. Jeff Smith for carefully polishing both language and style of this paper. 


\section{References}

Axford, W. I., McKenzie, J. F.: 1992, The origin of high speed solar wind streams. In: Marsch, E., Schwenn, R. (eds.) Solar Wind Seven, COSPAR Colloq. 3, Pergamon Press, 1-5.

Bale, S. D., Kellogg, P. J., Mozer, F. S., Horbury, T. S., Reme, H.: 2005, Measurement of the electric fluctuation spectrum of magnetohydrodynamic turbulence. Phys. Rev. Lett. 94, 215002.

Barnes, A., Hollweg, J. V.: 1974, Large-amplitude hydromagnetic waves. J. Geophys. Res. 79, $2302-2318$.

Chandran, B. D.: 2008, Weakly turbulent magnetohydrodynamic waves in compressible low- $\beta$ plasmas. Phys. Rev. Lett. 101, 269903.

Coles, W. A.: 1978, Interplanetary scintillation. Space Sci. Rev. 21, 411-425.

Cranmer, S. R., Kohl, J. L., Noci, G., Antonucci, E., Tondello, G., Huber, M. C. E., et al.: 1999, An empirical model of a polar coronal hole at solar minimum. Astrophys. J. 511, 481-501.

Cranmer, S. R., van Ballegooijen, A. A.: 2003, Alfvénic turbulence in the extended solar corona: Kinetic effects and proton heating. Astrophys. J. 594, 573-591.

Dasso, S., Milano, L. J., Matthaeus, W. H., Smith, C. W.: 2005, Anisotropy in fast and slow solar wind fluctuations. Astrophys. J. Lett. 635, L181-L184.

Gary, S. P.: 1993, Theory of Space Plasma Microinstabilities. Cambridge University Press, $\mathrm{UK}$.

Gary, S. P., Nishimura, K.: 2004, Kinetic Alfvén waves: Linear theory and a particle-in-cell simulation. J. Geophys. Res. 109, A02109.

Gary, S. P., Borovsky, J. E.: 2004, Alfvén-cyclotron fluctuations: Linear Vlasov theory. J. Geophys. Res. 109, A06105.

Gary, S. P., Smith, W.: 2009, Short-wavelength turbulence in the solar wind: Linear theory of whistler and kinetic Alfvén fluctuations. J. Geophys. Res. 114, A12105.

Gogoberidze G., Mahajan, S. M., Poedts, S.: 2009, Weak and strong regimes of incompressible magnetohydrodynamic turbulence. Phys. Plasmas 16, 072304.

Goldreich, P., Sridhar, S.: 1995, Toward a theory of interstellar turbulence. II: strong Alfvén turbulence. Astrophys. J. 438, 763-775.

Goldstein, B. E., Neugebauer, M., Zhang, L. D., Gary, S. P.: 2000, Observed constraint on proton-proton relative velocities in the solar wind. Geophys. Res. Lett. 27, 53-56.

He, J., Marsch, E., Tu, C., Yao, S., Tian, H.: 2011, Possible evidence of Alfvén-cyclotron waves in the angle distribution of magnetic helicity of solar wind turbulence. Astrophys. J. 731, 85.

Hollweg, J. V., Isenberg, P. A.: 2002, Generation of the fast solar wind: a review with emphasis on the resonant cyclotron interaction. J. Geophys. Res. 107, 1147.

Horbury, T. S., Forman, M. A., Oughton, S.: 2005, Spacecraft observations of solar wind turbulence: an overview. J. Plasma Phys. Controlled Fusion 47, B703.

Isenberg, P. A.: 2001, The kinetic shell model of coronal heating and acceleration by ion cyclotron waves 2: Inward and outward propagating waves. J. Geophys. Res. 106, 2924929260.

Kohl, J. L., Esser, R., Gardner, L. D., Habbal, S., Daigneau, P. S., Dennis, E. F., et al.: 1995, The Ultraviolet Coronagraph Spectrometer for the Solar and Heliospheric Observatory. Solar Phys. 162, 313-356.

Kohl, J. L., Noci, G., Antonucci, E., Tondello, G., Huber, M. C. E., Cranmer, S. R., et al.: 1998, UVCS/SOHO empirical determinations of anisotropic velocity distributions in the solar corona. Astrophys. J. 501, L127-L131.

Leamon, R. J., Smith, C. W., Ness, N. F., Matthaeus, W. H., Wong, H. K.: 1998, Observational constraints on the dynamics of the interplanetary magnetic field dissipation range. $J$. Geophys. Res. 103, 4775-4787.

Li, X., Habbal, S. R.: 2001, Damping of fast and ion cyclotron oblique waves in the multi-ion fast solar wind. J. Geophys. Res. 106(A6), 10669-10680.

Li, X., Habbal, S. R., Kohl, J. L., Noci, G. C.: 1998, The effect of temperature anisotropy on observations of Doppler dimming and pumping in the inner corona. Astrophys. J. 501, L133-L137.

Li, X., Lu, Q., Chen, Y., Li, B., Xia, L.: 2010, A kinetic Alfvén wave and the proton distribution function in the fast solar wind. Astrophys. J. Lett. 719, L190-L193.

MacBride, B. T., Smith, C. W., Vasquez, B. J.: 2010, Inertial-range anisotropies in the solar wind from 0.3 to 1 AU: Helios 1 observations. J. Geophys. Res. 115, A07105. 
Markovskii, S. A., Hollweg, J. V.: 2004, Intermittent heating of the solar corona by heat flux-generated ion cyclotron waves. Astrophys. J. 609, 1112-1122.

Markovskii, S. A., Vasquez, B. J., Chandran, B. D. G.: 2010, Perpendicular proton heating due to energy cascade of fast magnetosonic waves in the solar corona. Astrophys. J. 709, 1003-1008.

Marsch, E., Tu, C. Y.: 2001, Heating and acceleration of coronal ions interacting with plasma waves through cyclotron and Landau resonance. J. Geophys. Res. 106, 227-238.

Marsch, E., Rosenbauer, H., Schwenn, R., Muehlhaeuser, K. H., Neubauer, F. M.: 1982, Solar wind helium ions - observations of the Helios solar probes between 0.3 and 1 AU. J. Geophys. Res. 87, 35-51.

Matthaeus, W. H., Goldstein, M. L., Roberts, D. A.: 1990, Evidence for the presence of quasitwo-dimensional nearly incompressible fluctuations in the solar wind. J. Geophys. Res. 95, 20673-20683.

Neugebauer, M., Snyder, C. W.: 1962, Solar plasma experiment. Science 138, 1095-1097.

Neugebauer, M., Goldstein, B. E., Smith, E. J., Feldman, W. C.: 1996, Ulysses observations of differential alpha-proton streaming in the solar wind. J. Geophys. Res. 101, 17047-17055.

Osmane, A., Hamza, A. M., Meziane, K.: 2010, On the generation of proton beams in fast solar wind in the presence of obliquely propagating Alfvén waves. J. Geophys. Res. 115, A05101.

Parker, E. N.: 1958, Dynamics of the interplanetary gas and magnetic fields. Astrophys. J. 128, 664-676.

Perez, J. C., Boldyrev, S.: 2008, On weak and strong magnetohydrodynamic turbulence. Astrophys. J. Lett. 672, L61-L64.

Podesta, J. J., Borovsky, J. E., Gary, S. P.: 2010, A kinetic Alfvén wave cascade subject to collisionless damping cannot reach electron scales in the solar wind at 1 AU. Astrophys. J. $\mathbf{7 1 2}, 685$.

Sahraoui, F., Goldstein, M. L., Robert, P., Khotyaintsev, Y. V.: 2009, Evidence of a cascade and dissipation of solar-wind turbulence at the electron gyroscale. Phys. Rev. Lett. 102, 231102 .

Sahraoui, F., Goldstein, M. L., Belmont, G., Canu, P., Rezeau, L.: 2010, Three dimensional anisotropic $k$ spectra of turbulence at subproton scales in the solar wind. Phys. Rev. Lett. 105, 131101

Smith, C. W., Mullan, D. J., Ness, N. F., Skoug, R. M., Steinberg, J.: 2001, Day the solar wind almost disappeared: Magnetic field fluctuations wave refraction and dissipation. $J$. Geophys. Res. 106, 18625-18634.

Stix, T. H.: 1992, Waves in Plasmas. Springer, 237-304.

Wicks, R. T., Horbury, T. S., Chen, C. H. K., Schekochihin, A. A.: 2011, Anisotropy of imbalanced Alfvén turbulence in fast solar wind. Phys. Rev. Lett. 106, 045001. 
Table 1. Symbols used in this paper and their denotations

\begin{tabular}{|c|c|}
\hline Symbol & Description \\
\hline$c$ & light speed \\
\hline$e$ & an electron charge \\
\hline$k_{\mathrm{B}}$ & Boltzmann constant \\
\hline$x, y, z$ & Cartesian coordinate system \\
\hline $\mathbf{B}_{\mathbf{0}}, B_{0}$ & uniform background magnetic field along $z$-axis $\left(B_{0}=\left|\mathbf{B}_{\mathbf{0}}\right|\right)$ \\
\hline$\delta \mathbf{B}$ & magnetic field turbulence \\
\hline$\delta \mathbf{E}$ & electric field turbulence \\
\hline$\|$ & parallel direction ( $z$-axis) \\
\hline$\perp$ & perpendicular direction ( $x y$-plane) \\
\hline$P$ & polarization of electric field fluctuation in $x y$-plane \\
\hline,+- & left- and right-handed electric field polarizations in $x y$-plane \\
\hline $\begin{array}{l}\mathbf{e}_{E} \\
\mathbf{e}_{E}^{+}, \mathbf{e}_{E}^{-}\end{array}$ & $\begin{array}{l}\text { unimodular electric field polarization, and } \\
\text { its left- and right-handed components }\end{array}$ \\
\hline $\mathbf{k}, k, e_{k}$ & wave vector $(k=|\mathbf{k}|)$ and its unimodular vector $\left(e_{k}=\mathbf{k} / k\right)$ \\
\hline$k_{\|}, k_{\perp}$ & parallel and perpendicular components of $\mathbf{k}$ \\
\hline$\theta$ & $\begin{array}{l}\text { angle between the propagation and the ambient magnetic field } \\
\theta=\arctan \left(k_{\perp} / k_{\|}\right)\end{array}$ \\
\hline$j(\alpha, \mathrm{p}, \mathrm{e})$ & plasma species $j$ in an alpha/proton/electron $(\alpha / \mathrm{p} / \mathrm{e})$ plasma \\
\hline$m_{j}\left(m_{\alpha}, m_{\mathrm{p}}, m_{\mathrm{e}}\right)$ & mass of species $j$ \\
\hline$z_{j}\left(z_{\alpha}, z_{\mathrm{p}}, z_{\mathrm{e}}\right)$ & electric charge number of species $j \quad\left(z_{\alpha}=2, \quad z_{\mathrm{p}}=z_{\mathrm{e}}=1\right)$ \\
\hline$n_{j}\left(n_{\alpha}, n_{\mathrm{p}}, n_{\mathrm{e}}\right)$ & $\begin{array}{l}\text { abundance of species } j \\
\text { (neutral charge: } n_{\alpha} z_{\alpha}+n_{\mathrm{p}} z_{\mathrm{p}}+n_{\mathrm{e}} z_{\mathrm{e}}=0 \text { ) } \\
\text { (minor heavy ion: } n_{\alpha} \ll n_{\mathrm{p}} \text { ) }\end{array}$ \\
\hline$\omega_{\mathrm{p} j} \quad\left(\omega_{\mathrm{p} \alpha}, \omega_{\mathrm{pp}}, \omega_{\mathrm{pe}}\right)$ & plasma frequency $\omega_{\mathrm{p} j}=\sqrt{4 \pi n_{j} z_{j}^{2} e^{2} / m_{j}}$ \\
\hline $\begin{array}{l}\Omega_{j} \\
\left(\Omega_{\alpha}, \Omega_{\mathrm{p}}, \Omega_{\mathrm{e}}\right)\end{array}$ & $\begin{array}{l}\text { cyclotron frequency } \Omega_{j}=z_{j} \text { e } B_{0} /\left(m_{j} c\right) \\
\left(\Omega_{\mathrm{p}}=2 \Omega_{\alpha}\right)\end{array}$ \\
\hline$\omega, \omega_{\mathrm{r}}, \omega_{\mathrm{i}}$ & complex wave frequency as well as its real and imaginary parts \\
\hline$v_{\mathrm{A}}$ & Alfvén speed $v_{\mathrm{A}}=B_{0} / \sqrt{4 \pi n_{\mathrm{p}} m_{\mathrm{p}}}$ \\
\hline $\begin{array}{l}U_{\| j} \\
\left(U_{\| \alpha}, U_{\| \mathrm{e}}\right)\end{array}$ & $\begin{array}{l}\text { differential speed of species } j \text { relative to protons along } \mathbf{B}_{\mathbf{0}} \\
\text { (zero current: } n_{\alpha} z_{\alpha} U_{\| \alpha}+n_{\mathrm{e}} z_{\mathrm{e}} U_{\| \mathrm{e}}=0 \text { ) }\end{array}$ \\
\hline$T_{\| j}\left(T_{\| \alpha}, T_{\| \mathrm{p}}, T_{\| \mathrm{e}}\right)$ & parallel temperature of species $j$ \\
\hline$T_{\perp j}\left(T_{\perp \alpha}, T_{\perp \mathrm{p}}, T_{\perp \mathrm{e}}\right)$ & perpendicular temperature of species $j$ \\
\hline$\gamma_{j}\left(\gamma_{\alpha}, \gamma_{\mathrm{p}}, \gamma_{\mathrm{e}}\right)$ & temperature anisotropy $\gamma_{j}=T_{\perp j} / T_{\| j}$ \\
\hline $\begin{array}{l}\beta_{j} \\
\left(\beta_{\alpha}, \beta_{\mathrm{p}}, \beta_{\mathrm{e}}\right)\end{array}$ & $\begin{array}{l}\text { species beta defined as } \\
\text { a ratio of species } j \text { 's thermal pressure to magnetic pressure } \\
\beta_{j}=8 \pi n_{j} k_{\mathrm{B}} T_{\| j} / B_{0}^{2}\end{array}$ \\
\hline$\beta$ & plasma beta $\left(\beta=\beta_{\alpha}+\beta_{\mathrm{p}}+\beta_{\mathrm{e}}\right)$ \\
\hline
\end{tabular}


Table 1.

\begin{tabular}{ll}
\hline$v_{\| j}\left(v_{\| \alpha}, v_{\| \mathrm{p}}, v_{\| \mathrm{e}}\right)$ & parallel thermal speed $v_{\| j}=\sqrt{2 k_{\mathrm{B}} T_{\| j} / m_{j}}$ \\
\hline$v_{\perp j}\left(v_{\perp \alpha}, v_{\perp \mathrm{p}}, v_{\perp \mathrm{e}}\right)$ & perpendicular thermal speed $v_{\perp j}=\sqrt{2 k_{\mathrm{B}} T_{\perp j} / m_{j}}$ \\
\hline$f_{j}\left(f_{\alpha}, f_{\mathrm{p}}, f_{\mathrm{e}}\right)$ & $\begin{array}{l}\text { gyrotropic velocity distribution function of species } j \\
\text { in terms of } v_{\| j} \text { and } v_{\perp j}\end{array}$ \\
\hline$\xi_{j}\left(\xi_{\alpha}, \xi_{\mathrm{p}}, \xi_{\mathrm{e}}\right)$ & arbitrary-order general resonance factor of species $j$ \\
\hline$\xi_{j}^{0}\left(\xi_{\alpha}^{0}, \xi_{\mathrm{p}}^{0}, \xi_{\mathrm{e}}^{0}\right)$ & zero-order Landau resonance factor of species $j$ \\
\hline$\xi_{j}^{+}\left(\xi_{\alpha}^{+}, \xi_{\mathrm{p}}^{+}, \xi_{\mathrm{e}}^{+}\right)$ & first-order cyclotron resonance factor of species $j$ \\
\hline$a_{\| j}\left(a_{\| \alpha}, a_{\| \mathrm{p}}, a_{\| \mathrm{e}}\right)$ & normalized parallel acceleration rate of species $j$ \\
\hline$Q_{\| j}\left(Q_{\| \alpha}, Q_{\| \mathrm{p}}, Q_{\| \mathrm{e}}\right)$ & normalized parallel heating rate of species $j$ \\
\hline$Q_{\perp j}\left(Q_{\perp \alpha}, Q_{\perp \mathrm{p}}\right)$ & normalized perpendicular heating rate of specie $j$ \\
\hline
\end{tabular}

Table 2. Fixed prescription among all initial plasma parameters

\begin{tabular}{ll}
\hline Symbol & Value \\
\hline parallel temperature ratio & $4: 1: 1$ \\
$T_{\| \alpha}: T_{\| \mathrm{p}}: T_{\| \mathrm{e}}$ & \\
\hline temperature anisotropy of alpha particles $\gamma_{\alpha}$ & 1 \\
\hline temperature anisotropy of protons $\gamma_{\mathrm{p}}$ & 1 \\
\hline temperature anisotropy of electrons $\gamma_{\mathrm{e}}$ & 1 \\
\hline
\end{tabular}

Table 3. Parametric study of initial plasma parameters

\begin{tabular}{llllll}
\hline Section & Figure & $\begin{array}{l}\text { Propagation } \\
\text { angle } \theta\end{array}$ & $\begin{array}{l}\text { Density ratio } \\
n_{\alpha}: n_{\mathrm{p}}\end{array}$ & $\begin{array}{l}\text { Differential } \\
\text { speed of alpha } \\
\text { particles } U_{\| \alpha}\end{array}$ & $\begin{array}{l}\text { Proton beta } \\
\beta_{\mathrm{p}}\end{array}$ \\
\hline 3,4 & $1-8$ & $0^{\circ}-89^{\circ}$ & 0.02 & 0 & 0.01 \\
\hline 5 & - & $0^{\circ}-89^{\circ}$ & 0 & 0 & 0.01 \\
\hline 6 & 9,10 & $60^{\circ}$ & 0.02 & $0-1 v_{\mathrm{A}}$ & 0.01 \\
\hline 7 & - & $60^{\circ}$ & 0.02 & 0 & $0.001-10$ \\
\hline
\end{tabular}




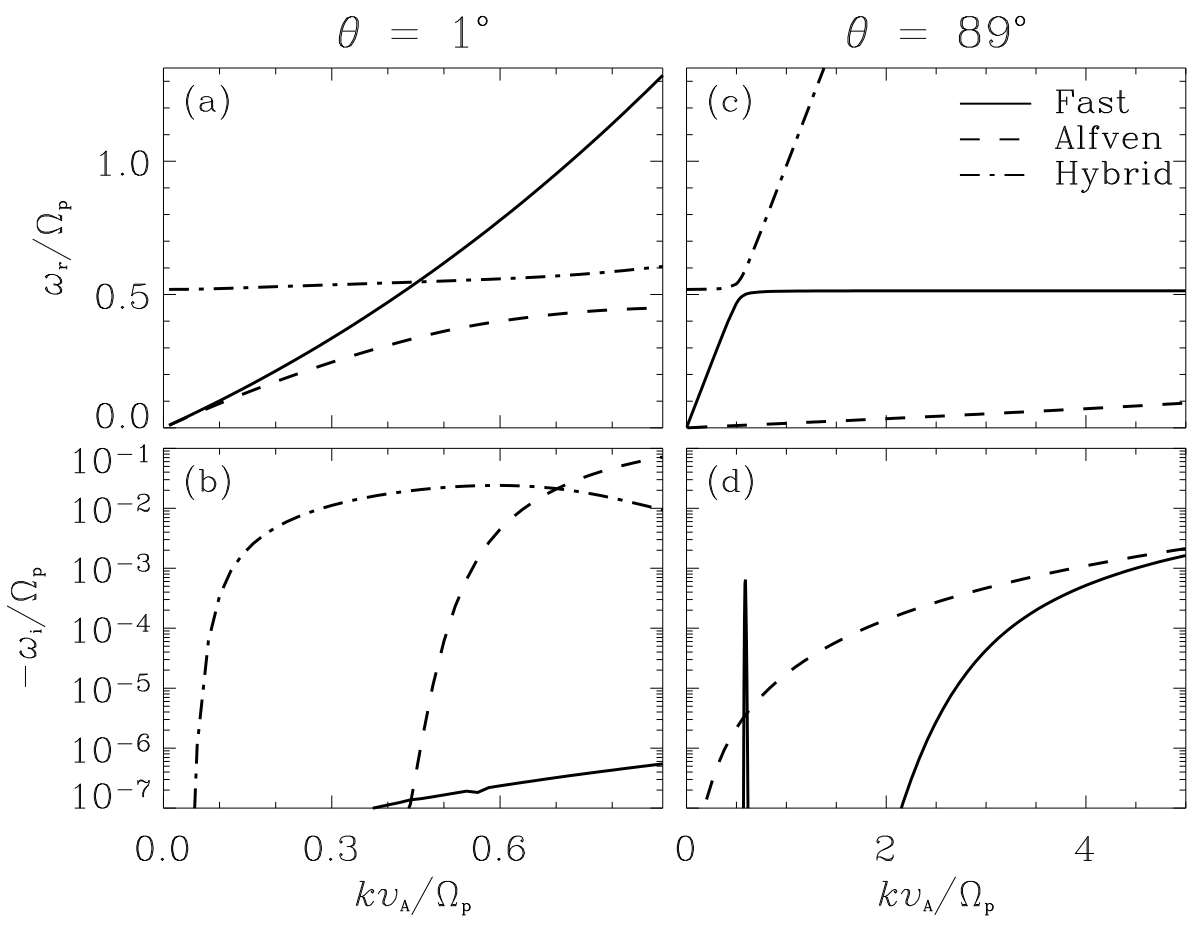

Figure 1. Dispersion relations of Alfvén, fast, and hybrid alpha-proton cyclotron waves at $(\mathrm{a}, \mathrm{b}) \theta=1^{\circ}$ and $(\mathrm{c}, \mathrm{d}) \theta=89^{\circ}$ in a proton/alpha/electron plasma. Note that linear mode coupling between hybrid and fast waves happens in panel (c). 


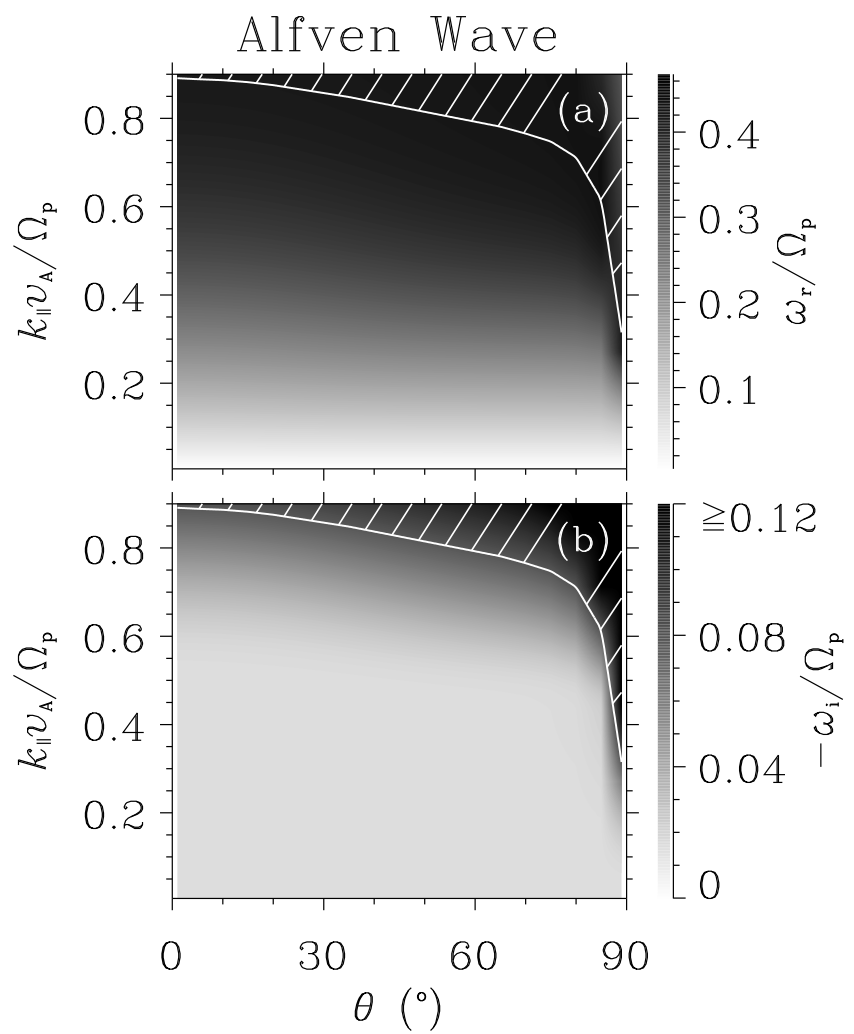

Figure 2. For Alfvén waves, the dependence of complex frequency $\left(\omega=\omega_{\mathrm{r}}+i \omega_{\mathrm{i}}\right)$ on the parallel wave number $k_{\|}$and propagation angle $\theta$. Heavily damped waves satisfying $\omega_{\mathrm{i}}(\mathbf{k})<-\frac{\omega_{\mathrm{r}}(\mathbf{k})}{2 \pi}$ are indicated by a hatched region. 


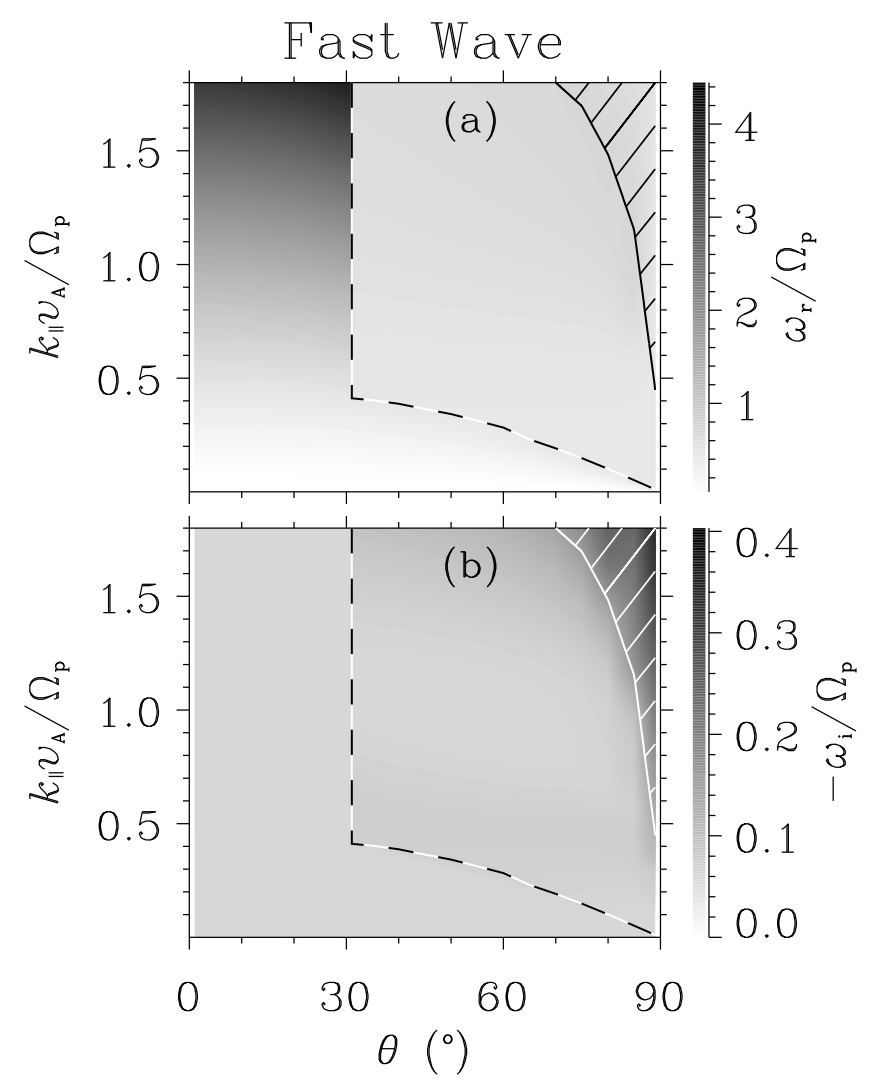

Figure 3. For Fast waves, the dependence of complex frequency $\left(\omega=\omega_{\mathrm{r}}+i \omega_{\mathrm{i}}\right)$ on the parallel wave number $k_{\|}$and propagation angle $\theta$. Linear mode conversion occurs for $\theta \geq 31^{\circ}$, with its position in the $k_{\|}-\theta$ domain denoted by a black-and-white line. 


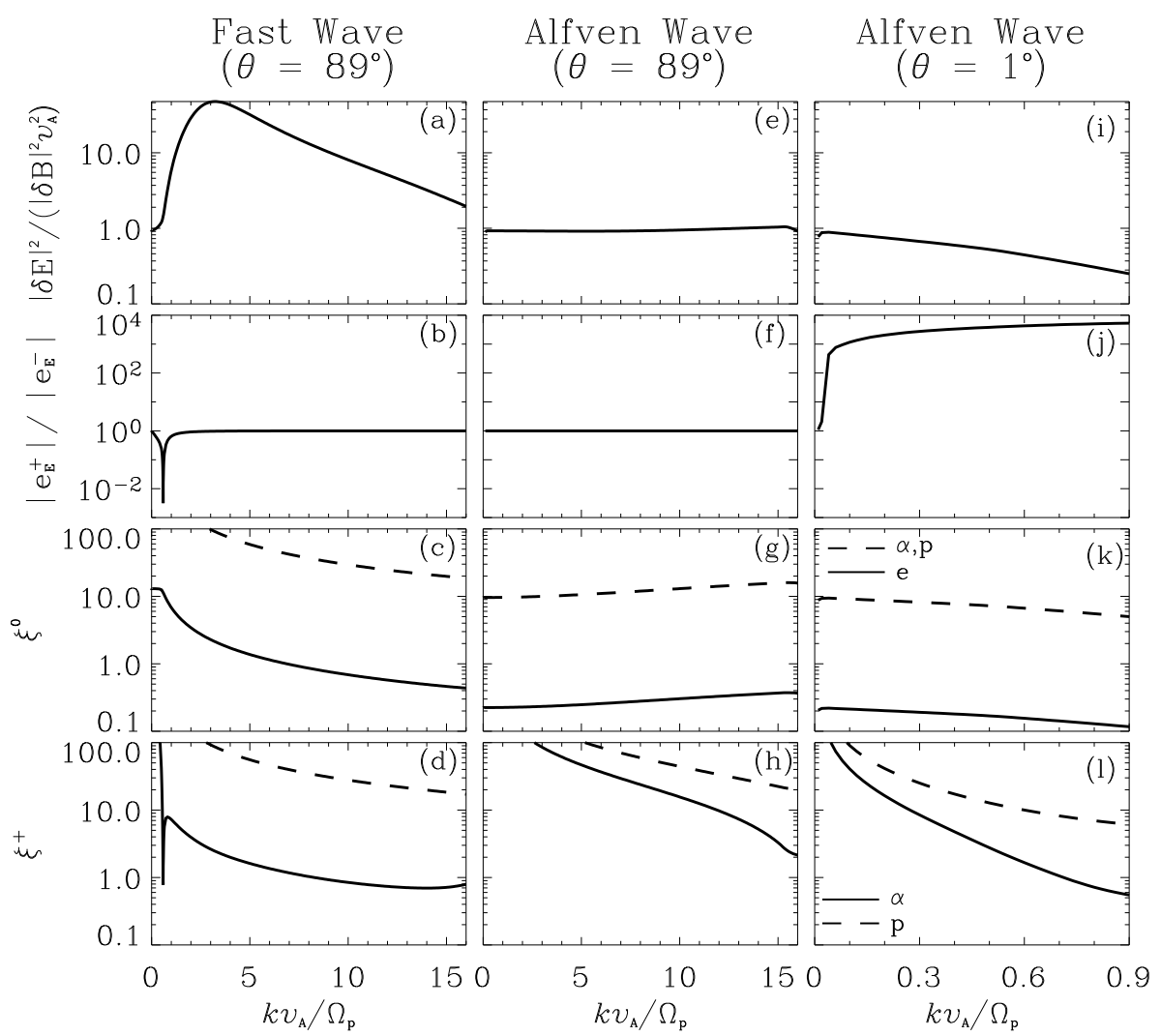

Figure 4. Properties of quasi-perpendicular fast waves, quasi-perpendicular and quasi-parallel Alfvén waves in a proton/alpha/electron plasma: (a,e,i) ratio of electric to magnetic field turbulence spectra $|\delta \mathbf{E}|^{2} /|\delta \mathbf{B}|^{2}$, (b,f,j) ratio of left- to right-handed polarizations for electric field spectrum $\left|\mathbf{e}_{E}^{+}\right| /\left|\mathbf{e}_{E}^{-}\right|,(\mathrm{c}, \mathrm{g}, \mathrm{k})$ Landau resonance factors $\xi^{0}$, and $(\mathrm{d}, \mathrm{h}, \mathrm{l})$ cyclotron resonance factors $\xi^{+}$. 


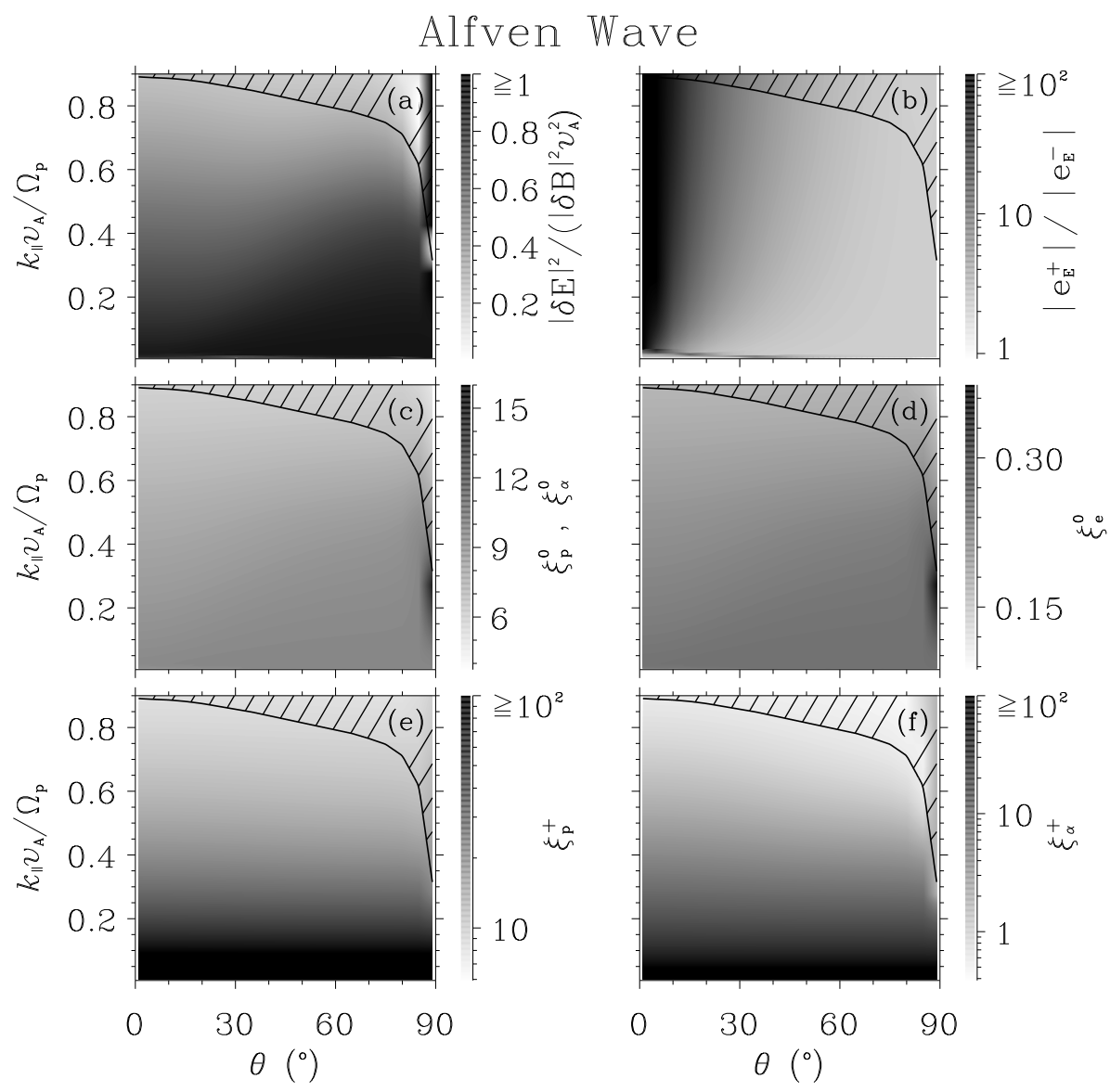

Figure 5. The dependence of Alfvén wave properties in a proton/alpha/electron plasma on the parallel wave number $k_{\|}$and propagation angle $\theta$ : (a) ratio of electric to magnetic field turbulence spectra $|\delta \mathbf{E}|^{2} /|\delta \mathbf{B}|^{2}$, (b) ratio of left- to right-handed polarizations for electric field spectrum $\left|\mathbf{e}_{E}^{+}\right| /\left|\mathbf{e}_{E}^{-}\right|$, (c) Landau resonance factors of protons $\xi_{\mathrm{p}}^{0}$ and alpha particles $\xi_{\alpha}^{0}$, (d) Landau resonance factor of electrons $\xi_{\mathrm{e}}^{0}$, (e) cyclotron resonance factor of protons $\xi_{\mathrm{p}}^{+}$, and (f) cyclotron resonance factor of alpha particles $\xi_{\alpha}^{+}$. 
Fast Wave
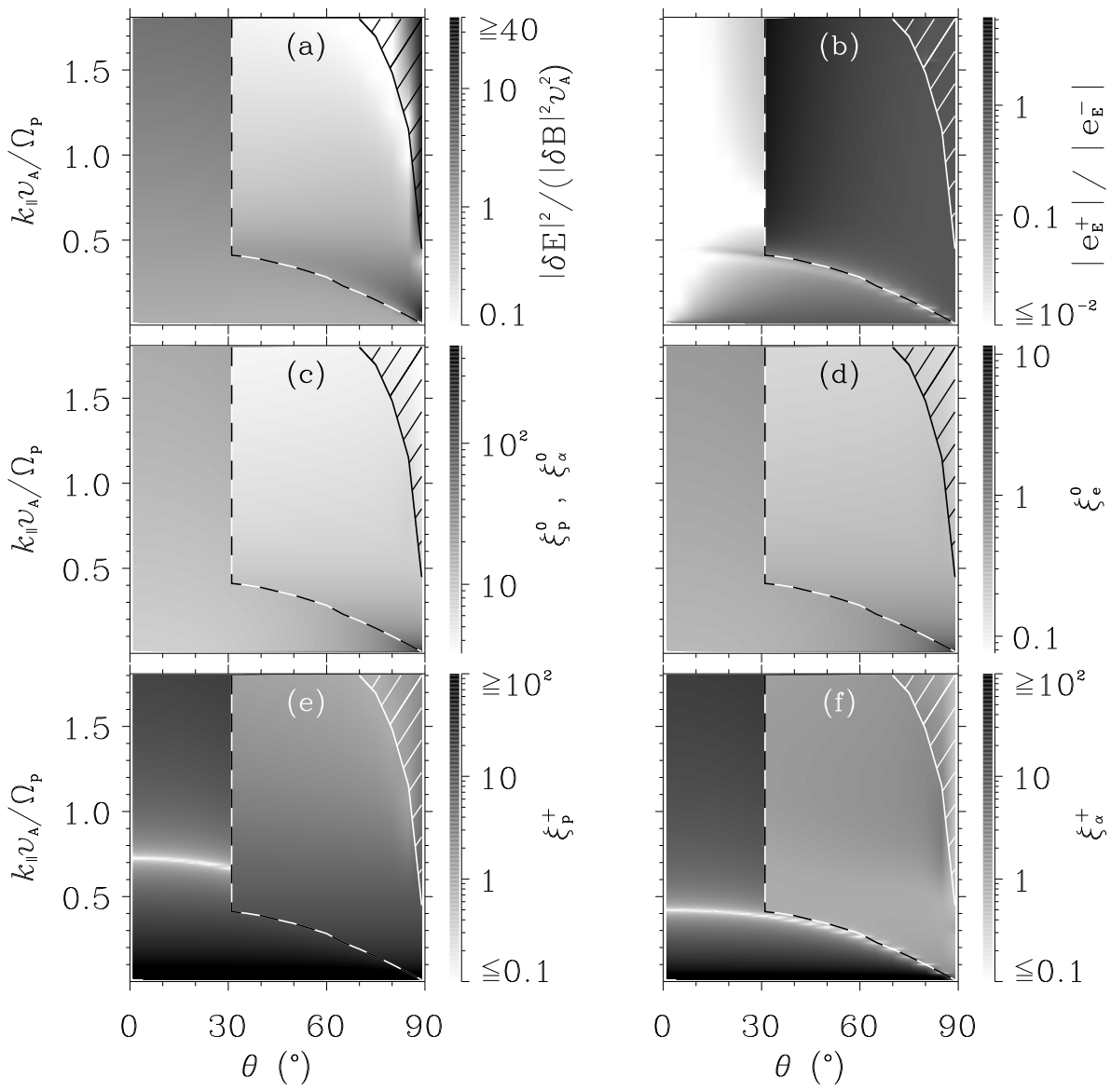

Figure 6. The dependence of fast wave parameters in a proton/alpha/electron plasma on the parallel wave number $k_{\|}$and propagation angle $\theta$ : (a) ratio of electric to magnetic field turbulence spectra $|\delta \mathbf{E}|^{2} /|\delta \mathbf{B}|^{2}$, (b) ratio of left- to right-handed polarizations for electric field spectrum $\left|\mathbf{e}_{E}^{+}\right| /\left|\mathbf{e}_{E}^{-}\right|$, (c) Landau resonance factors of protons $\xi_{\mathrm{p}}^{0}$ and alpha particles $\xi_{\alpha}^{0}$, (d) Landau resonance factor of electrons $\xi_{\mathrm{e}}^{0}$, (e) cyclotron resonance factor of protons $\xi_{\mathrm{p}}^{+}$, and (f) cyclotron resonance factor of alpha particles $\xi_{\alpha}^{+}$. 


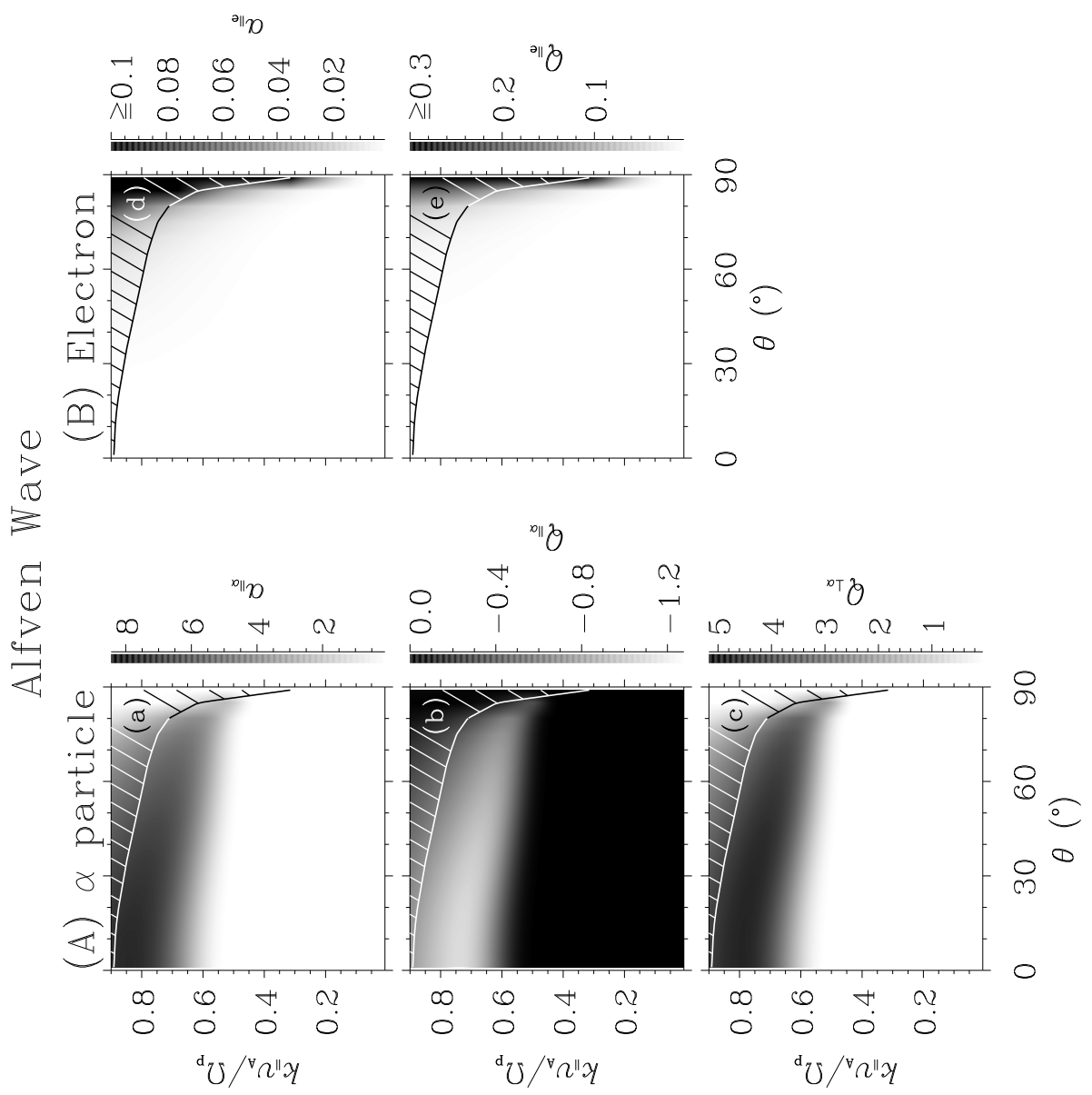

Figure 7. The energization capabilities of Alfvén waves in a proton/alpha/electron plasma on (A) alpha particles and (B) electrons. From top to bottom are (a,d) parallel acceleration $a_{\|}$, (b,e) parallel heating $Q_{\|}$, and (c) perpendicular heating $Q_{\perp}$ over the $k_{\|}-\theta$ domain. Quantities of $a_{\|}, Q_{\|}$, and $Q_{\perp}$ are already normalized by the wave spectrum $|\delta \mathbf{B}(\mathbf{k})|^{2}$. Here the proton energization and the perpendicular electron heating are essentially zero. 

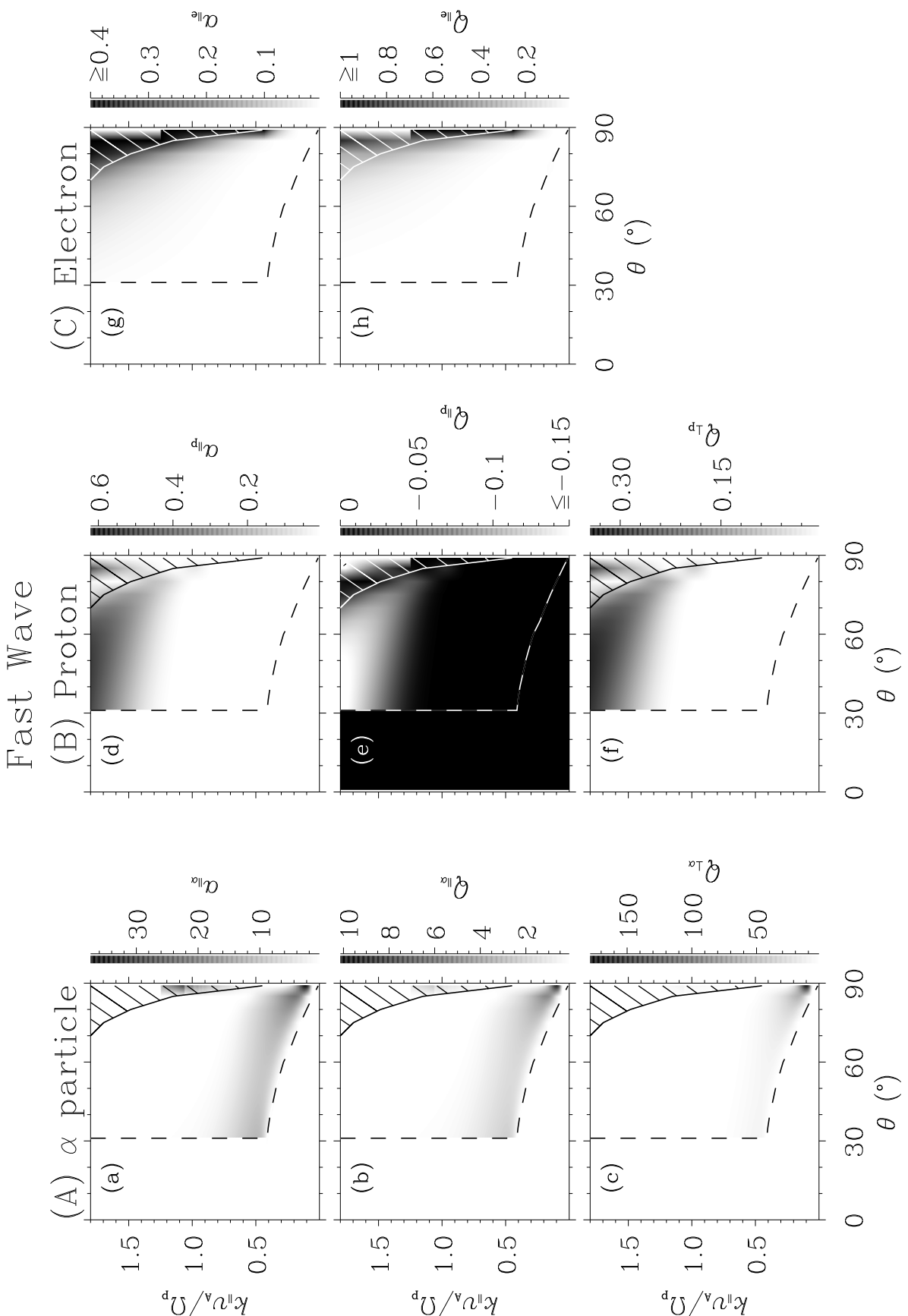

Figure 8. The energization effects of fast waves in a proton/alpha/electron plasma on (A) alpha particles, (B) protons, (C) electrons. From top to bottom are (a,d,g) parallel acceleration, $(\mathrm{b}, \mathrm{e}, \mathrm{h})$ parallel heating, and $(\mathrm{c}, \mathrm{f})$ perpendicular heating over the $k_{\|}-\theta$ domain. Here the perpendicular electron heating $Q_{\perp \mathrm{e}}$ is essentially zero. 

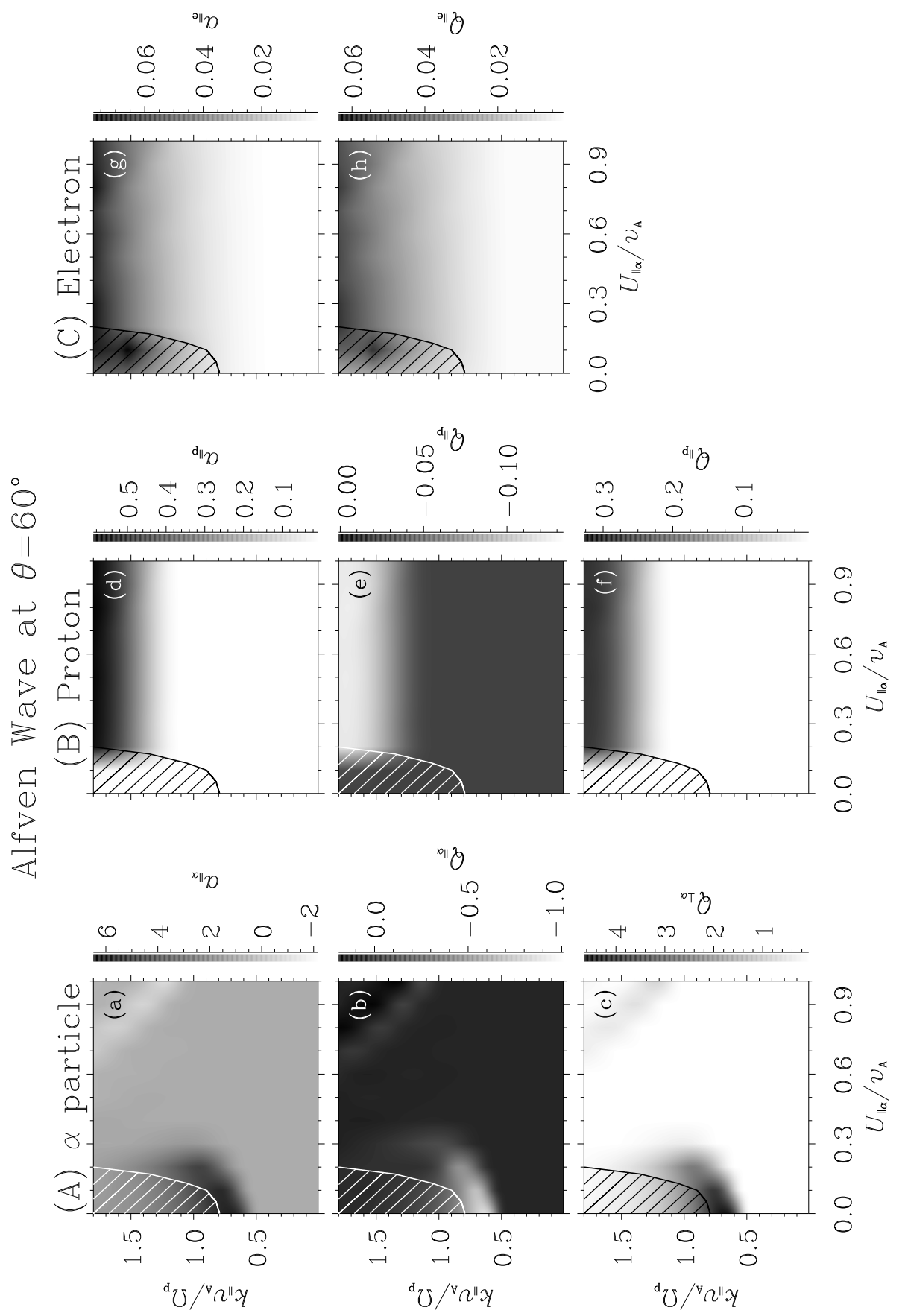

Figure 9. For Alfvén waves at propagation angle $\theta=60^{\circ}$, differential speed of alpha particles $U_{\| \alpha}$ in a proton/alpha/electron plasma results in different energizations of (A) alpha particles, (B) protons, and (C) electrons. 

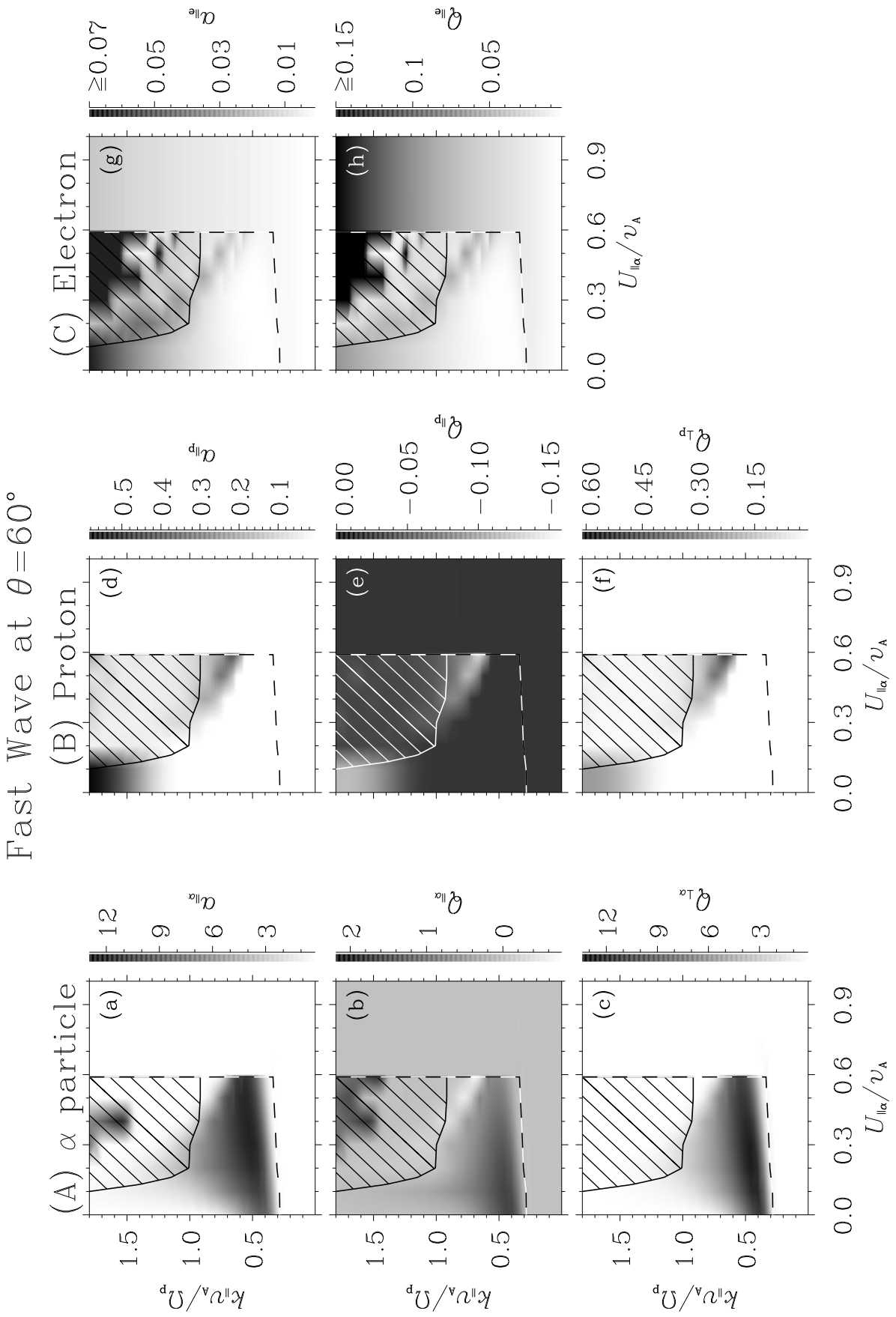

Figure 10. Depending on the differential speed of alpha particles $U_{\| \alpha}$ in a proton/alpha/electron plasma, the energization consequence of fast waves at propagation angle $\theta=60^{\circ}$. Linear mode conversion occurs at $U_{\| \alpha}<0.6 v_{\mathrm{A}}$, denoted as a black-and-white line in the $k_{\|}-U_{\| \alpha}$ domain. 
SOLA: SoPh2011Rev2.tex; 2 August 2018; 21:42; p. 30 